Article

\title{
A Survey of the Brazilian Dicranocentrus Schött (Collembola, Orchesellidae, Heteromurini) with the Description of a New Species and Notes on the Genus
}

\author{
Bruno C. Bellini ${ }^{1, *(D)}$, Nikolas G. Cipola ${ }^{2}$ and Orquianne J. R. Siqueira ${ }^{1, *}$ \\ 1 Laboratório de Collembola, Departamento de Botânica e Zoologia, Centro de Biociências, \\ Universidade Federal do Rio Grande do Norte-UFRN, BR 101, Lagoa Nova, Campus Universitário, \\ Natal 59072-970, Brazil \\ 2 Laboratório de Sistemática e Ecologia de Invertebrados do Solo, Instituto Nacional de Pesquisas da \\ Amazônia-INPA, COBIO/Entomologia, Campus II, Petrópolis, Manaus 69067-375, Brazil; \\ nikolasgc@gmail.com \\ * Correspondence: entobellini@gmail.com (B.C.B.); orquianne@gmail.com (O.J.R.S.)
}

Received: 15 September 2020; Accepted: 27 September 2020; Published: 16 October 2020

Simple Summary: Springtails (Collembola) are microarthropods commonly found in terrestrial habitats, especially associated to the soil. Dicranocentrus Schött is one of the most representative genera of the family Orchesellidae Börner of springtails, with 69 described species in tropical regions of the world. Here we describe a new Brazilian species of the genus, especially using data on its chaetotaxy, the comparative study of the shape and the arrangement of body chaetae. We also use such data to compare and better understand the morphology of other species of the genus, and to better recognize them using comparative tables and identification keys.

Abstract: Dicranocentrus Schött is the most diverse and widespread taxon of Neotropical Orchesellidae. In Brazil, the genus is represented by 11 species found in humid forested areas of Atlantic and Amazon forests domains. Here we describe in detail Dicranocentrus abestado sp. nov. from Chapada Diamantina, Caatinga domain, Brazil. The new species belongs to the marias group sensu Mari-Mutt, due to the absence of most posterior macrochaetae on the dorsal head, and resembles other Neotropical species with 3, 2 and 2 central macrochaetae on abdominal segments I-III. However, the new species is unique especially by its reduced colour pattern combined with its empodial complex morphology. We compare Dicranocentrus abestado sp. nov. with 27 other taxa from the New and Old World, including all species with 3 macrochaetae on the first abdominal segment; provide notes and details on the morphology of the compared species plus identification keys to Brazilian and all species of the genus with similar abdominal chaetotaxy. We also discuss the current taxonomical knowledge on Brazilian Dicranocentrus and provide notes on its chaetotaxy and Heteromurinae systematics.

Keywords: chaetotaxy; Entomobryoidea; Heteromurinae; identification keys; review; taxonomy

\section{Introduction}

Entomobryoidea Womersley, 1934 [1] is the largest superfamily of springtails, with more than 2500 described species [2,3]. The superfamily comprises about $28 \%$ of all known Collembola species, and is currently subdivided into three families: Orchesellidae Börner, 1906 [4], Entomobryidae Schäffer, 1896 [5] and Paronellidae Börner, 1906 [4] and sensu Zhang et al., 2019 [2]. In Brazil the Entomobryoidea are represented by 159 species, about $36 \%$ of all known Brazilian springtails, distributed in all three families and 23 genera [6], of which the family Orchesellidae are represented by two subfamilies, four tribes, five genera and 17 species, summarized in Table 1. 
Dicranocentrus Schött, 1893 [7] has a Pantropical distribution and is the second largest genus of Orchesellidae, with 69 nominal species, just after Orchesella Templeton, 1836 [8], with almost 100 species [3]. Dicranocentrus is the most widespread and species-rich genus of Neotropical Orchesellidae [9-11]. Its species are epiedaphic, mostly found on ground, over litter, moss, rooting wood and even associated to epiphytes, being rarely found inside termites' nests $[12,13]$. The genus' main diagnostic features are antennae with six segments (antennal segments I and II subdivided), 8 eyes, prelabral chaetae simple (not bifurcate), ungues with a single outer tooth and dental spines, if present, simple (a detailed diagnosis is presented in the results topic) $[12,14,15]$. Currently, there are 11 species of Dicranocentrus known from Brazil, found in humid forested areas of Atlantic and Amazon forests domains (Table 1) [6,13,16-21].

Table 1. List of Brazilian species of Orchesellidae Börner, 1906 sensu Zhang et al. [2] and their known distribution.

\begin{tabular}{|c|c|}
\hline Taxa & Known Distribution in Brazil (Biomes) \\
\hline \multicolumn{2}{|l|}{ Heteromurini Absolon and Ksenemann, 1942 [22] } \\
\hline H. anae Cipola, $2016 *[14]$ & Amazon Forest \\
\hline \multicolumn{2}{|l|}{ Dicranocentrus Schött, 1893 [7] } \\
\hline D. albicephalus Xisto and Mendonça, 2017* [21] & Atlantic Forest \\
\hline D. bicolor Handschin, $1924^{*, * * *}[13]$ & Atlantic Forest \\
\hline D. cuprum Xisto and Mendonça, $2016 *[20]$ & Atlantic Forest \\
\hline D. heloisae Arlé and Mendonça, 1982 * [16] & Atlantic Forest \\
\hline D. magnus Xisto and Mendonça, 2017 * [21] & Atlantic Forest \\
\hline D. marimutti Xisto and Mendonça, $2017 *$ [21] & Atlantic Forest \\
\hline \multicolumn{2}{|l|}{ Mastigocerini Mari-Mutt, 1980 [25] } \\
\hline \multicolumn{2}{|l|}{ Mastigoceras Handschin, 1924 * [13] } \\
\hline M. camponoti Handschin, 1924 *** [13] & Atlantic Forest, Amazon Forest, Caatinga \\
\hline \multicolumn{2}{|l|}{ Nothobryinae Zhang and Deharveng, 2015 [26] } \\
\hline \multicolumn{2}{|l|}{ Nothobryini Soto-Adames et al., 2008 [27]sensu Nunes et al. 2020 [28] } \\
\hline \multicolumn{2}{|l|}{ Nothobrya Arlé, 1961 * [29] } \\
\hline N. arlei Silveira and Mendonça, 2016 *[30] & Atlantic Forest \\
\hline N. schubarti Arlé, 1961* [29] & Cerrado, Caatinga \\
\hline N. sertaneja Nunes and Bellini, $2019 *$ [31] & Caatinga \\
\hline \multicolumn{2}{|l|}{ Capbryini Nunes et al. 2020 [28] } \\
\hline \multicolumn{2}{|l|}{ Capbrya Barra, 1999 [32] } \\
\hline
\end{tabular}

Legends: [] description references; $\left({ }^{*}\right)$ endemic from Brazil; $\left({ }^{* *}\right)$ possibly a species complex; $\left.{ }^{(* *}\right)$ Species inquirenda (see the discussion topic); (?) unclear data.

Here, we describe in detail a new species of Brazilian Dicranocentrus and compare its affinities with 27 other species. We also provide an updated diagnosis to the genus, comparative tables of Brazilian species as well as species with similar abdominal chaetotaxy, discuss the current knowledge on Brazilian Dicranocentrus, and provide notes on the chaetotaxy of the genus and Heteromurinae systematics.

\section{Materials and Methods}

Specimens of the new species were preserved in $70 \%$ ethanol and sorted with a stereomicroscope Leica S8AP0 (Leica, Wetzlar, Germany). After, they were cleared in Nesbitt's solution, washed in Arlé's liquid and mounted on glass slides in Hoyer's medium, following the procedures described by Arlé and Mendonça [16] and Jordana et al. [33], with few adaptations. Glass slide specimens were studied with Leica DM500 (Leica, Wetzlar, Germany) and DM750 (Leica, Wetzlar, Germany) microscopes, both connected to drawing tubes. The habitus of the new species was photographed with stereomicroscope Nikon SMZ1500 (Nikon, Tokyo, Japan), with a Nikon DS-Ri1 camera (Nikon, Tokyo, 
Japan) attached, using NIS-Elements AR v.4.51.00 software (Nikon, Tokyo, Japan). Photographs and raw drawings were digitally improved in Corel Photo-Paint X8 (Corel, Ottawa, Canada) and CorelDraw X8 software (Corel, Ottawa, Canada), respectively.

The chaetotaxy terminology used in this study follows mainly Fjellberg [34] to labial palp papillae; Gisin [35] to labial chaetotaxy with additions of Zhang and Pan [36]; Cipola et al. [37] to labral chaetotaxy; Mari-Mutt [12] to dorsal head chaetotaxy with additions of Soto-Adames [38] and Zhang et al. [15]; Szeptycki [39] and Zhang and Deharveng [26] to S-chaetotaxy; an Szeptycki [40] to dorsal chaetotaxy, with additions and modifications provided by Soto-Adames [38], Cipola et al. [14] and Zhang et al. [2,41].

The abbreviations used in the descriptions are: Abd.-abdominal segment(s); Ant.-antennal segment(s); mac-macrochaeta(e); mes—-mesochaeta(e); mic-microchaeta(e); ms-S-microchaeta(e); sens—ordinary S-chaeta(e); and Th.-thoracic segment(s). Antennal segments I and II subdivisions are: "a" to proximal subarticle, " $\mathrm{b}$ " to distal one. Depository abbreviation: CC/UFRN—Collembola Collection of the Biosciences Center of Federal University of Rio Grande do Norte, Brazil; INPA-Invertebrate Collection of the National Institute of Amazonian Research, Manaus, Brazil.

The symbols used in the drawings to represent the dorsal chaetotaxy schemes are: large blank circles to mac; large black circles to mes; small black circles to mic; blank circles with small black circle inside to mac or mic; black chaetae-like drawings to sens or ms; black circles crossed by a line for pseudopores; bothriotricha-like drawings to bothriotricha; a dash above or under any symbol to chaetae present or absent. Taxonomic description and comparisons are based on half body. Chaetae labels (including rows) and labial papillae are marked in bold in the text.

\section{Results}

\subsection{Taxonomic Summary and Genus Diagnosis}

Order Entomobryomorpha Börner, 1913 [42]

Superfamily Entomobryoidea Womersley, 1934 [1]

Orchesellidae Börner, 1906 [4] sensu Zhang et al. 2019 [2]

Family Heteromurinae Absolon and Ksenemann, 1942 [22] sensu Zhang and Deharveng, 2015 [26]

Tribe Heteromurini Absolon and Ksenemann, 1942 [22] sensu Zhang et al. 2020 [15]

Genus Dicranocentrus Schött, 1893 [7]

Diagnosis: Specimens mostly pigmented, colour patterns variable, eyepatches black. Antennae six-segmented, Ant. I and II basally subdivided, proximal subarticles smaller than the distal ones, Ant. III usually longer than Ant. IV, rarely subequal or smaller; apical bulb of Ant. IV absent; Ant. III-IV annulated, devoid of scales. Postantennal organ absent. Eight eyes. Prelabral chaetae smooth and simple (not bifurcate). Labial chaetae e smooth; post-labial quadrangle (anterior chaetae surrounding the cephalic groove) chaetae smooth or rough, never ciliate. Th. II not projected over head. Abd. V with 4 or 7 sens (atypical). Ungues with a single outer tooth. Simple dental spines present or absent. Mucronal spine present. Data adapted and modified of Mari-Mutt [12], Cipola et al. [14], Zhang et al. [15] and Xisto and Mendonça [43].

Type species: Dicranocentrus gracilis Schött, 1893 [7]

Remarks: The presence of rounded and/or truncate coarsely ciliate scales on body and appendages, sens and $\mathbf{m s}$ formulae of dorsal Th. II to Abd. III = 2,2| 1,3,3 and 1,0| 1,0,1, respectively, short Abd. IV (less than two times the length of the Abd. III at the midline) and bidentate mucro were suppressed from the genus diagnosis since they are suprageneric diagnostic features. The updated diagnosis changes the number of sensilla on Abd. V compared to previous literature, as in $[2,14,15]$, due to the description of the new species. More details on Dicranocentrus overall morphology are presented in $[12,14,15,43-45]$. 
3.2. Dicranocentrus abestado sp. nov. Siqueira, Bellini, and Cipola

Figures 1-6, Tables 2 and 3

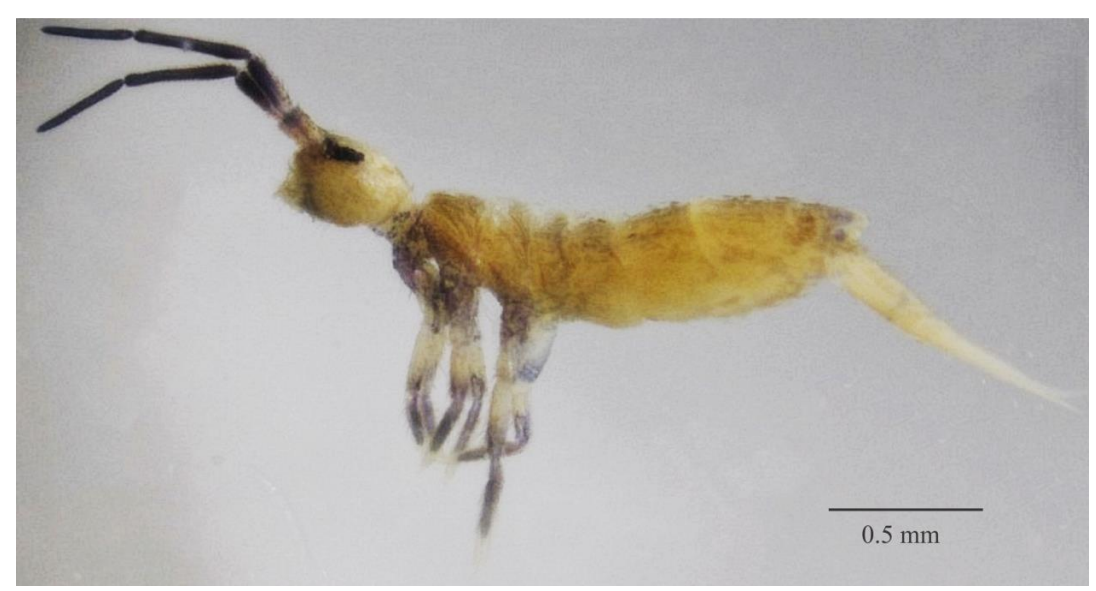

Figure 1. Dicranocentrus abestado sp. nov. habitus, specimen fixed in ethanol (lateral view).

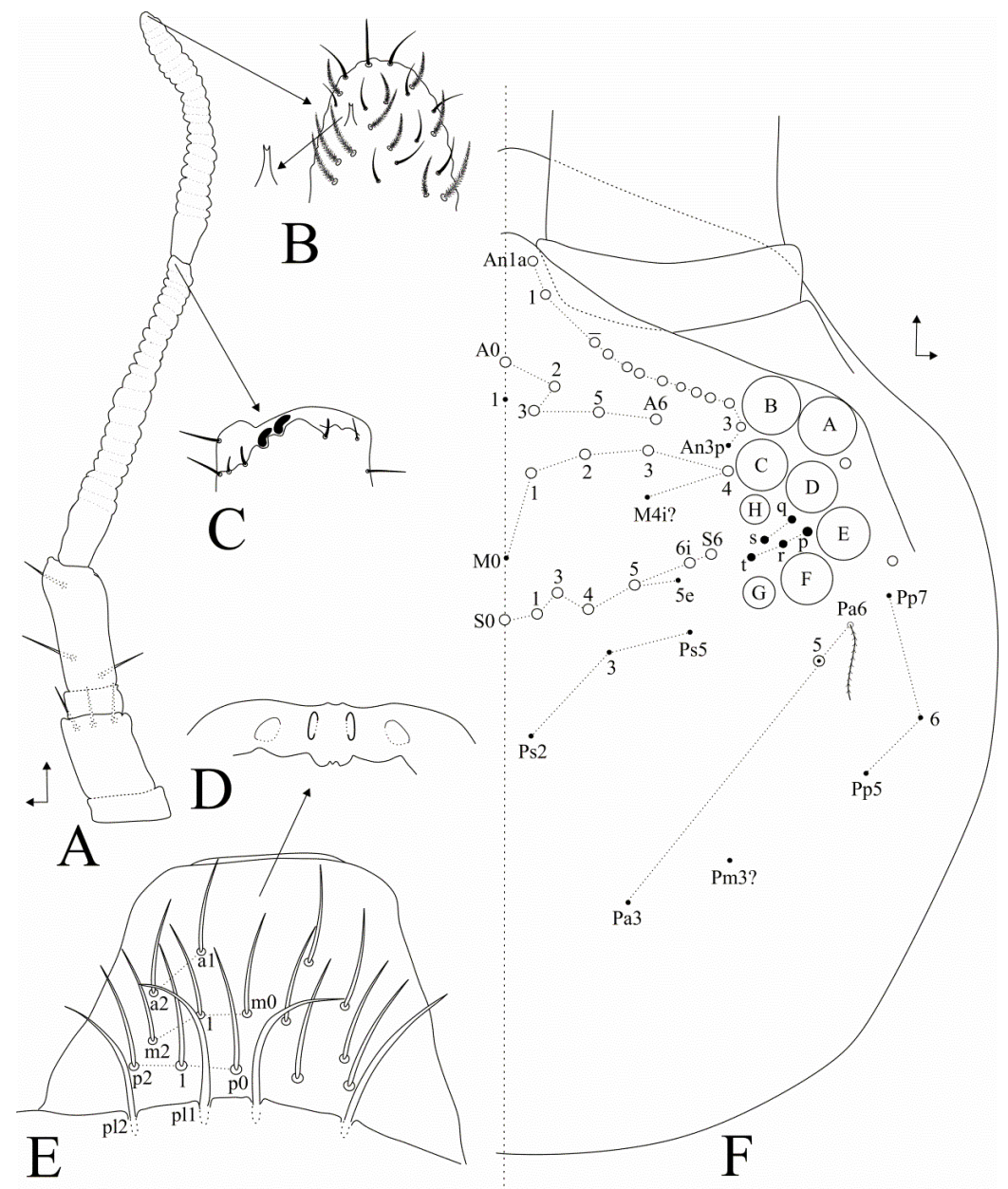

Figure 2. Dicranocentrus abestado sp. nov. head: (A) left antenna (dorsal view); (B) apical Ant. IV (ventro-lateral view), arrow indicates bifurcate pin projection; (C) apical organ of Ant. III (ventro-lateral view); (D) labral papillae; (E) labral and pre-labral chaetotaxy; (F) dorsal head chaetotaxy and eyes (right side). 


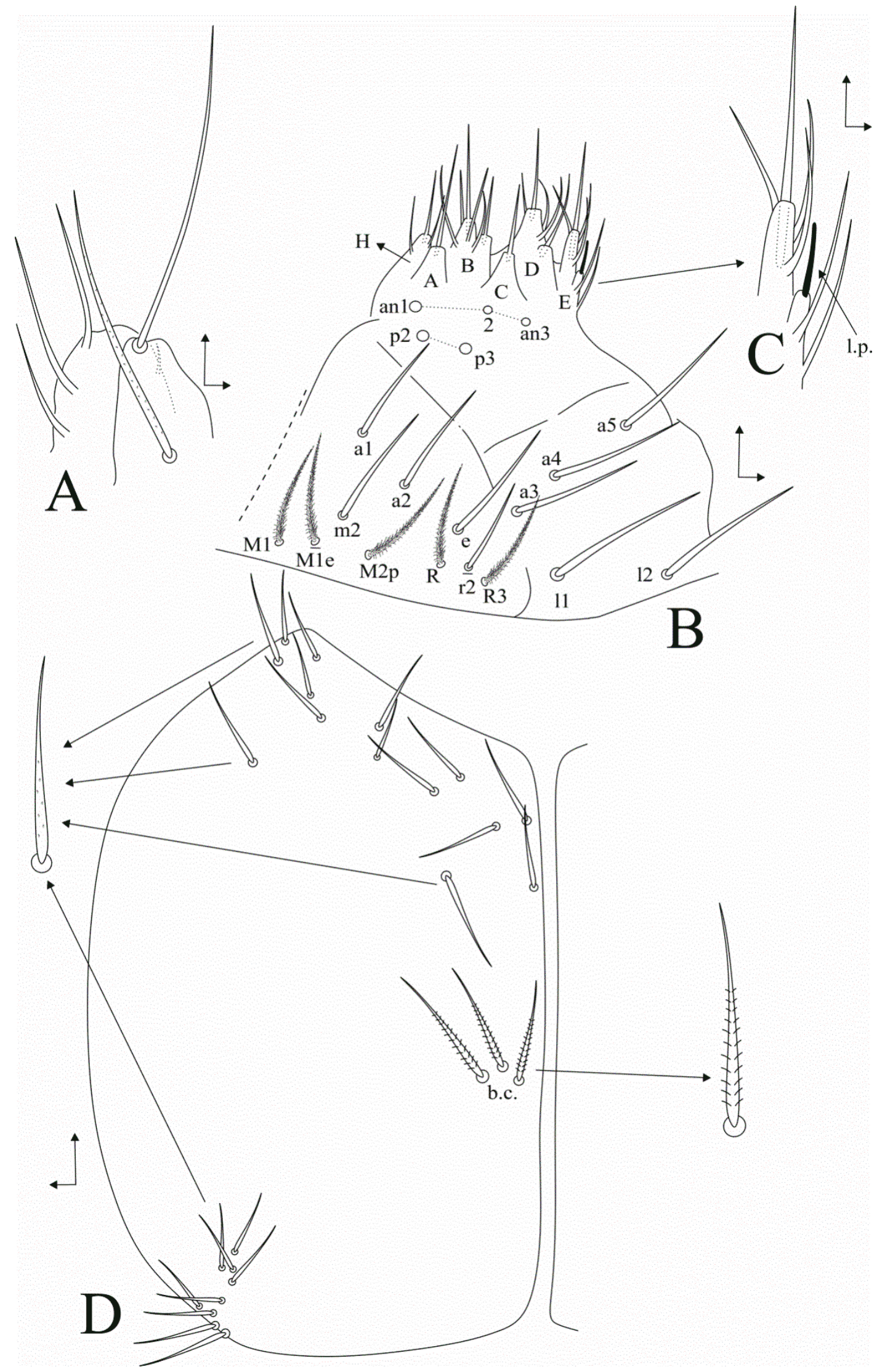

Figure 3. Dicranocentrus abestado sp. nov. ventral head: (A) maxillary outer lobe and sublobal plate (right side); (B) labium (right side); (C) labial papilla E (right side), arrow indicates lateral process (1.p.); (D) post-labial chaetotaxy (left side), arrows indicate morphology of chaetae, (b.c.) = basal chaetae. 


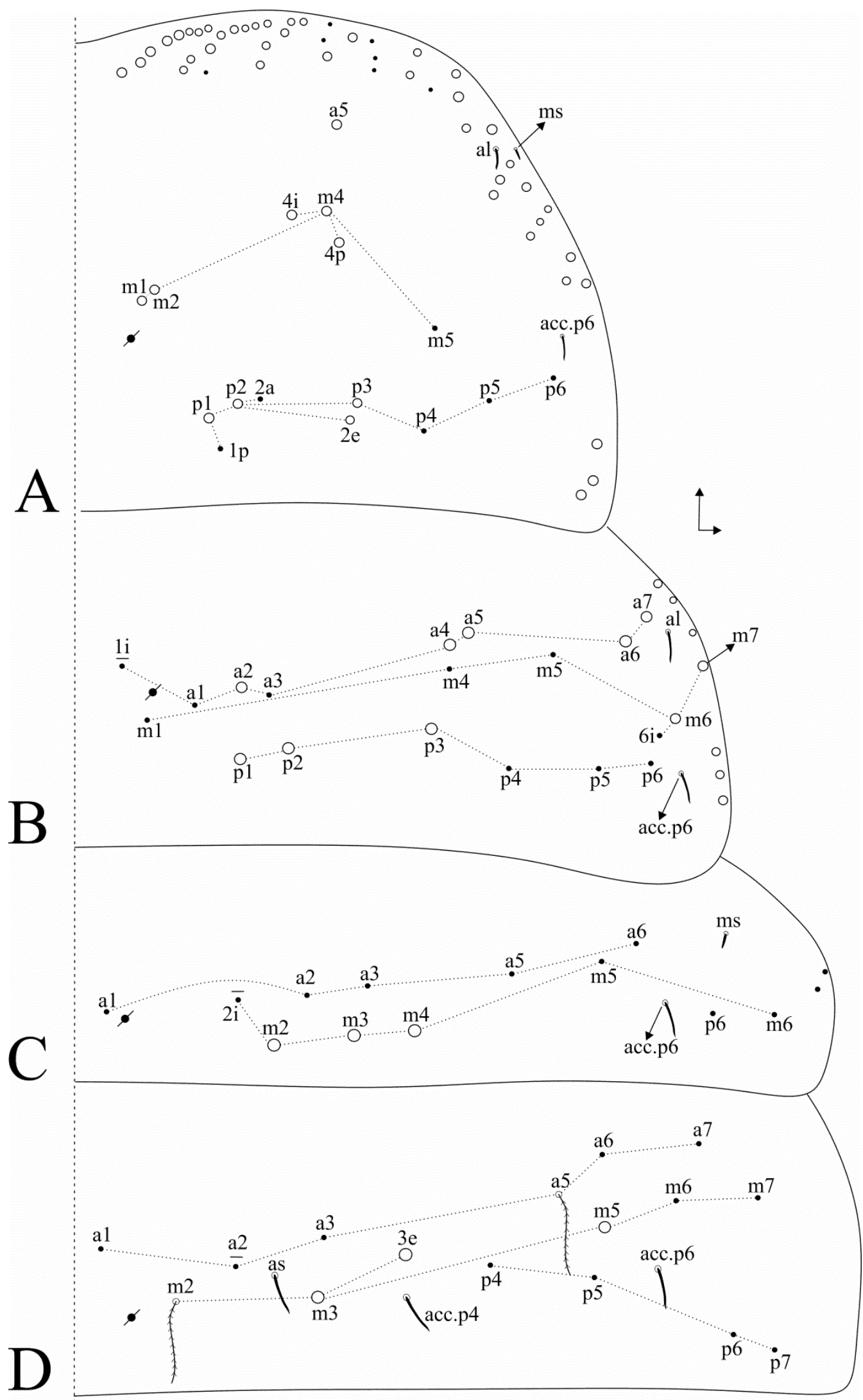

Figure 4. Dicranocentrus abestado sp. nov. trunk dorsal chaetotaxy (right side): (A) Th. II; (B) Th. III; (C) Abd. I; (D) Abd. II. 


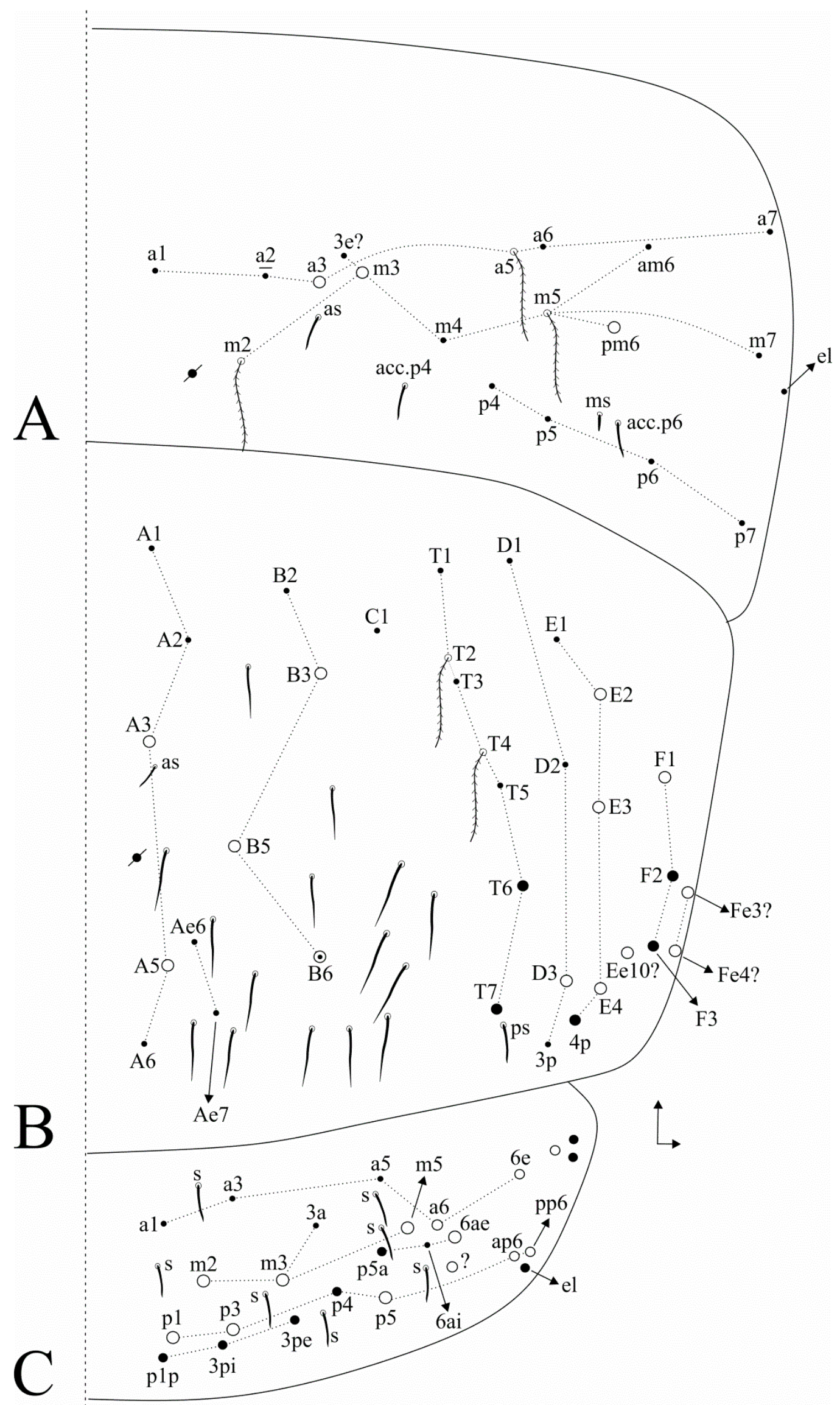

Figure 5. Dicranocentrus abestado sp. nov. trunk dorsal chaetotaxy (right side): (A) Abd. III; (B) Abd. IV; (C) Abd. V. 


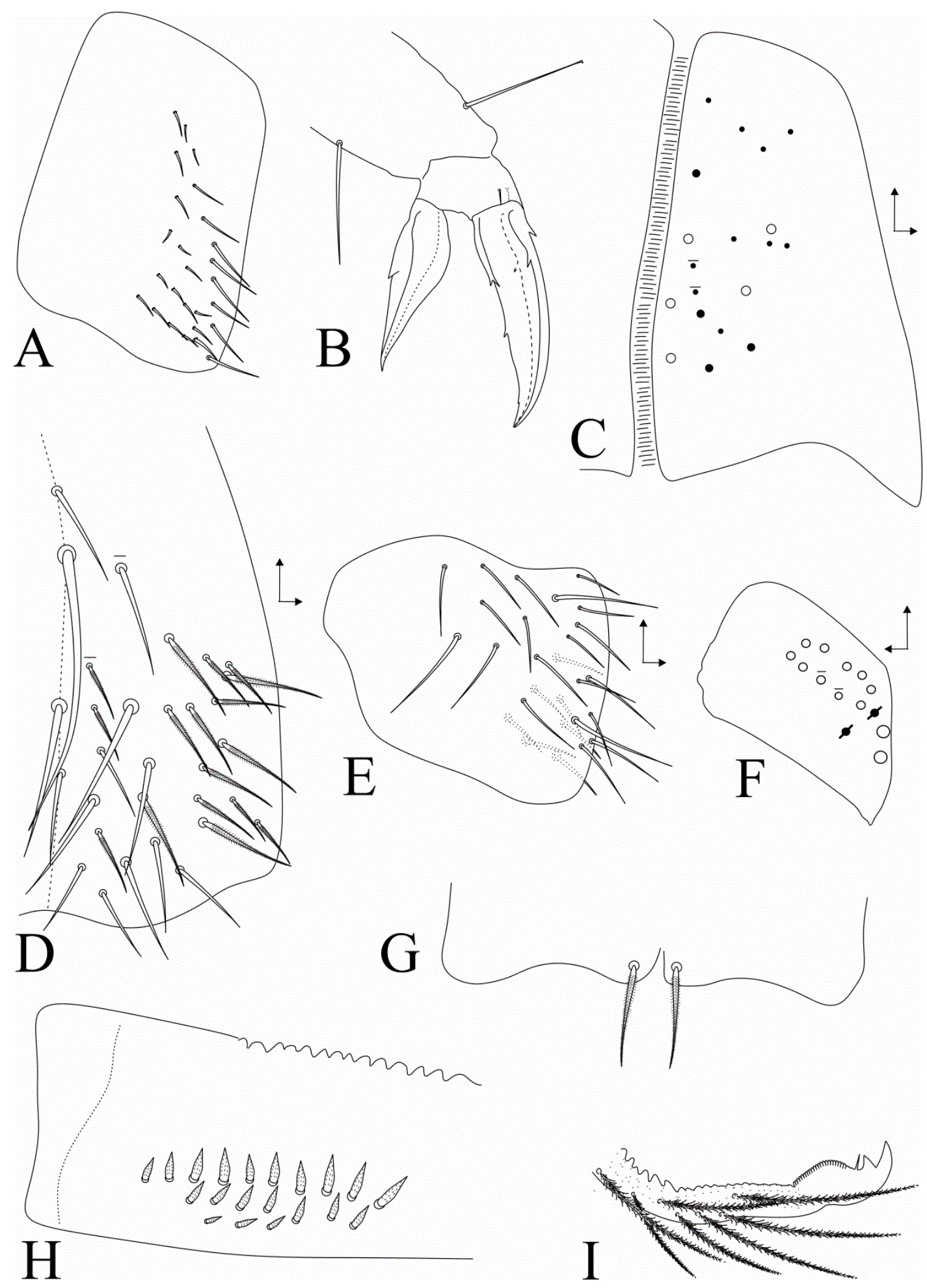

Figure 6. Dicranocentrus abestado sp. nov. trunk appendages: (A) trochanteral organ; (B) empodial complex of leg. III (anterior view); (C) ventral tube anterior face (right side); (D) ventral tube posterior face (right side); (E) ventral tube lateral flap (right side); (F) manubrial plate (left side); (G) manubrium ventro-distal chaetae; (H) spines on basal dens (internal view of right side); (I) distal dens and mucro.

Type material: Holotype: Female in slide (CC/UFRN): Brazil, Bahia State, Chapada Diamantina National Park, Lençóis Municipality ( $\left(12^{\circ} 33^{\prime} 47^{\prime \prime} \mathrm{S}, 41^{\circ} 23^{\prime} 28^{\prime \prime} \mathrm{W}\right)$, Caatinga phytogeographic domain, 
07.xi.2013, entomological aspirator, B.C. Bellini coll. Five paratypes in slides (CC/UFRN): two males, one female and two juveniles, plus one female (INPA-CLL 0000114), same data as holotype.

Diagnosis: Bluish pigments on Ant. II-IV, Th. II-III laterally to coxae, Abd VI, distal femurs and tibiotarsi. Ant. III longer than Ant. IV, Ant. IIb and Ib with three ventral smooth acuminate chaetae each. Labral papillae rounded. Dorsal head An, A, M, S and Ps series with 11-12, 5, 4, 7 and 0 mac, respectively; 0-1 posterior mac (Pa5 present or absent), five interocular chaetae. Maxillary outer lobe basal chaeta acuminate, labial papilla $\mathbf{E}$ lateral process (1.p.) finger-shaped, not reaching the papilla apex. Labial chaetae $\mathbf{m} \mathbf{2}, \mathbf{r} 2$ (if present) and $\mathbf{1 2}$ smooth, M1-1e (if present), M2p, R and R3 ciliate, labium unscaled. Three weakly ciliate post-labial basal chaetae (b.c.). Th. II with five $\mathbf{m}$ and four $\mathbf{p}$ main mac, $\mathbf{p} 5$ as mic; Th. III with 3, 0,3 central mac on rows $\mathbf{a}, \mathbf{m}$ and $\mathbf{p}$, respectively; Abd. I with three central mac; Abd. III with two central and one lateral mac; Abd. IV with 4-5 central mac, B6 as mic or mac; Abd. V with seven sens. Trochanteral organ with 22-27 chaetae. Tibiotarsi I-II with 3, tibiotarsus III with five distal smooth large chaetae on inner side, respectively. Tenent hairs weakly capitate or acuminate; ungues with one tiny apical tooth; unguiculi lanceolate, leaf shaped, antero-internal lamella wide, postero-external with one tooth. Ventral tube anterior face distally with one mac and two mes, posterior face with four unpaired plus 9-10 paired smooth chaetae, lateral flap with about 27 smooth chaetae. Tenaculum corpus with one rough chaeta. Manubrium with three and dens proximally with one dorsal smooth acuminate chaetae, manubrial plate with 10-12 ciliate chaetae plus two pseudopores, manubrium ventro-apical region with one ciliate chaeta. Dens with 16-20 spines distributed in 2-3 rows in proximo-internal region.

Description: Habitus typical of the genus (Figure 1). Body length (head + trunk) of holotype $=1.57 \mathrm{~mm}$; range of type series length (adult specimens) $=1.48-1.72 \mathrm{~mm}$; average body length of adult females $=1.59 \mathrm{~mm}$; average body length of adult males $=1.51 \mathrm{~mm}$; average body length of adults $=1.56 \mathrm{~mm}$. Specimens fixed in ethanol with yellowish background, Ant. I distally and Ant. II-IV dark blue, frontal head (clypeal and anterior post-labial regions), lateral Th. I-III, dorsal Abd. VI, coxae (including epi and subcoxae), distal half of femurs and proximal 3/4 of tibiotarsi light blue (Figure 1). Coarsely ciliate scales present on Ant. I, Ant. IIa, head (dorsally and ventrally), dorsal trunk, legs (all segments), ventral tube anterior and posterior faces, ventral manubrium and ventral dens. Dorsal head and trunk mac, mes and mic ciliate.

Head (Figures 2 and 3). Antennae shorter than trunk (Figures 1 and 2A), ratio antennae: Body $=1: 1.59$ (holotype), average 1:1.81 (adult specimens); Ant. III longer than Ant. IV, antennal ratio Ant. Ia-IV of holotype $=1: 2.2: 1: 3.7: 9.5: 7.5$; antennal ratio of type series adults $=1: 2.2-5: 1-2: 3.7-7: 8.7-9.5: 6.7-17.3$. Ant. IV subapical organite not seen, apparently missing; Ant. IV with a ventral subapical bifurcated pin projection, plus sens and ciliate chaetae (Figure 2B). Ant. III sense organ with 2 enlarged sensory rods, three guard sensilla plus at least four surrounding acuminate sens (Figure $2 \mathrm{C}$ ). Ant. $\mathrm{IIb}$ and $\mathrm{Ib}$ with three ventral smooth acuminate chaetae each (Figure 2A). Labral papillae rounded, internal marked and more oval, external more discrete (Figure 2D); Labral formula with four (a1-2), 5 (m0-2), 5 (p0-2) smooth chaetae, $\mathbf{p 0} \mathbf{- 2}$ slightly longer than others, a chaetae slender, prelabral chaetae larger than the labral ones (Figure 2E). Eyepatches largest lenses A and B, C-F subequal, G-H smaller than the others, with five interocular mes (Figure 2F). Head dorsal chaetotaxy with antennal (An) row with 11-12 mac, anterior (A) row with five (A0, A2-3, A5-6), medial (M) row with four (M1-4); sutural (S) row with seven (S0-1, S3-6), and post-occipital anterior (Pa) row with 0-1 (Pa5 present or absent) mac (Figure 2F). Maxillary outer lobe basal chaeta acuminate, slender and rough, slightly smaller than the apical smooth one, ratio basal chaeta:apical chaeta of holotype $=1: 1.1$; sublobal plate with four chaeta-like appendages, lateral one reduced, interno-basal one smaller than the interno-apical ones (Figure 3A). Labium with five main papillae (A-E), with 0, 5, 0, 4, 5 guard chaetae, respectively, papilla $\mathbf{E}$ lateral process (1.p.) finger-shaped, not reaching the papilla apex (Figure 3B,C); five proximal chaetae, an2-3 slightly smaller than others (Figure 3B); labial basomedial (submentum or labial triangle) and basolateral (mentum) fields with chaetae a1-5, m2, e, r2 (present or absent), 11-2 smooth; M1-1e (present or absent), M2p, R and R3 ciliate; labium unscaled (Figure 3B). Post-labial chaetotaxy with 14 rough, almost smooth, anterior chaetae, 
post-labial quadrangle with two chaetae, three basal chaetae slightly ciliate, posterior head with about nine grouped rough chaetae (Figure 3D).

Trunk dorsal chaetotaxy (Figures 4 and 5). Th. II, excluding anterior collar, with one anterior (a5), five medial (m1-2, m4-4p) and four posterior (p1-3) mac, p5 as mic (Figure 4A). Th. III with five anterior (a2, a4-7), two medial (m6-7) and three posterior (p1-3) mac (Figure 4B). Abd. I with three medial (m2-4) mac (Figure 4C). Abd. II with three medial (m3-3e, m5) mac (Figure 4D). Abd. III with one anterior (a3) and two medial (m3, pm6) mac (Figure 5A). Abd. IV with 4-5 central (A3, A5, B3, B5-6, B6 as mac or mic) and eight lateral (D3, E2-4, Ee10?, F1, Fe3?-Fe4?) mac, as sens just under A3, at least 14 central long sens (possibly many more) (Figure 5B). Ratio Abd. III:IV of holotype 1:1.7, ratio Abd. III:IV of type series adults 1: 1.5-1.7. Abd. V macrochaetotaxy with two anterior (a6-6e), three medial (m2-3, m5), one postero-anterior (p6ae), five posterior (p1, p3, p5, ap6, pp6) plus one mac without clear homology (?), four internal and three lateral sens (Figure 5C). More details on idio and S-chaetotaxy are represented in Figures 4 and 5.

Legs (Figure 6A,B): Trochanteral organ with 22-27 spine-like smooth chaetae, 22 on holotype (Figure 6A). Distal halves of tibiotarsi I-II with three and tibiotarsus III with five smooth chaetae on inner side, respectively. Tenent hairs discretely capitate or acuminate, two small pretarsal chaetae present, tibiotarsus III distal smooth chaeta subequal in length to tenant hair (Figure 6B); empodial complex III ratio of smooth chaeta: tenent hair: unguiculus: unguis of holotype as 1:1:1.5:2.2. Ungues with an outer pair of lateral undeveloped teeth on proximal $1 / 4$, inner side with four teeth: two paired basal teeth on proximal $1 / 3$, one unpaired median tooth on proximal $3 / 5$ with the same size of the basal teeth, and a minute apical tooth on distal 1/8 easily overlooked due to size and position; unguiculi lanceolate, leaf shaped, antero-internal lamella wide, all lamellae smooth except postero-external lamella with a large tooth on proximal 1/2 (Figure $6 \mathrm{~B}$ ).

Abdominal appendages (Figure 6C-I): Ventral tube anterior face with about 17-19 ciliate chaetae, five mac and 12-14 slender chaetae with different sizes, distal region with one mac and two mes (Figure 6C); posterior face with four unpaired (one very large) and 9-10 paired smooth chaetae with different sizes, plus 3-4 central ciliate chaetae (lateral side with many more ciliate chaetae, partially represented in Figure 6D); lateral flap with about 27 smooth chaetae, three clearly longer than the others (Figure 6E). Tenaculum corpus with a single rough chaeta, each ramus with four teeth. Dorsal manubrium with three smooth acuminate chaetae, one at the base, one median and one subapical; manubrial plate with 10-12 ciliate chaetae ( 2 inner as mac) plus two pseudopores (Figure 6F); manubrium ventro-apical region with one ciliate chaeta (Figure 6G). Dorsal dens with one smooth acuminate chaeta; dens with 16-20 rough spines (18-20 in holotype) in 2-3 rows in proximo-internal region (Figure $6 \mathrm{H}$ ). Mucro apical tooth larger than the basal one, mucronal spine reaching the apex of the basal tooth (Figure 6I).

Etymology: "abestado" or "abestada" is a regional expression used in Northeastern Brazil which means "fool". The expression is commonly used between friends.

Habitat: The new species was found in Chapada Diamantina National Park, in the southern region of Caatinga phytogeographic domain, from moist rocky and sandy soil samples surrounded by forested

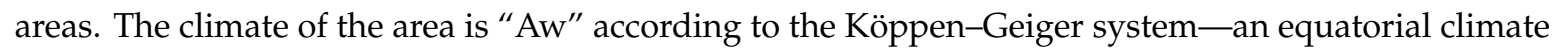
with dry desert-like summer [46]. The specimens were collected at the beginning of the raining season. 
Table 2. Comparison among Dicranocentrus species of the marias group from the New Word with similar morphology (head $\mathbf{S}$ series with 7 mac, basomedian labial field with M1 chaeta ciliate, $\mathbf{m} \mathbf{2}$ and $\mathbf{e}$ smooth, papilla E lateral process short, Th. II without p5 mac and Abd. I-III with 3, 2 and 2 central mac, respectively).

\begin{tabular}{|c|c|c|c|c|c|c|}
\hline & Snecios [References] & abestado & antillensis & icelosmarias & marias & paramoensis \\
\hline & & sp. nov. & {$[12,45]$} & [48] & {$[12,45,49]$} & [47] \\
\hline & Type Locality: & Chapada Diamantina & Camp Perrin, & Brick Kiln, & Las Marias, & Páramo de Mucubají, \\
\hline Characteristics & & Bahia, Brazil & Haiti & Saint Kitts and Nevis & Puerto Rico & Mérida, Venezuela \\
\hline \multicolumn{2}{|c|}{$\begin{array}{l}\text { Body bluish/violetish } \\
\text { Pigmentation on: }\end{array}$} & $\begin{array}{l}\text { Ant., anterior head, } \\
\text { lateral Th., dorsal Abd. } \\
\text { VI and part of legs }\end{array}$ & $\begin{array}{l}\text { Ant., anterior head, part } \\
\text { of legs, lateral Abd. I-IV } \\
\text { and posterior Abd. III-IV }\end{array}$ & Unpigmented ${ }^{* *}$ & $\begin{array}{l}\text { Ant., anterior head, } \\
\text { lateral trunk, legs or } \\
\text { entirely pigmented }\end{array}$ & $\begin{array}{l}\text { Entire body and appendages, } \\
\text { except furca }\end{array}$ \\
\hline Interocular chaetae & & 5 & ? & $3-4$ & ? & $?$ \\
\hline Dorsal head mac & Pp5 & - & - & $-?$ & - & + \\
\hline \multirow{5}{*}{$\begin{array}{l}\text { Basomedian and } \\
\text { basolateral labial } \\
\text { fields }\end{array}$} & $\mathbf{R}$ (smaller) & $\mathrm{C}$ & - & s & S & $\mathrm{S} / \mathrm{C}$ \\
\hline & L1 & S & $?$ & S & $?$ & $\mathrm{C}$ \\
\hline & L2 & S & $?$ & S & $?$ & C \\
\hline & extra chaetae* & $0-1 \mathrm{~S}, 2-3 \mathrm{C}$ & - & $3-4 \mathrm{~S}, 1 \mathrm{C}$ & $1-3 S, 1-2 C$ & $0-1 \mathrm{~S}, 6-7 \mathrm{C}$ \\
\hline & scales & - & + & ? & - & - \\
\hline \multirow{3}{*}{$\begin{array}{l}\text { Cephalic groove b.c. } \\
\text { Th. II mac }\end{array}$} & & 3 & ? & 3 & ? & 2 \\
\hline & $\mathrm{m} 4$ group & 3 & 2 & 3 & 3 & 2 \\
\hline & p row & 4 & 5 & 4 & 4 & 5 \\
\hline Th. III central mac & $\mathrm{a}, \mathrm{m}, \mathrm{p}$ rows & $3,0,3$ & $4,1,3$ & $3,0,3$ & $3,0,3$ & $2,1,3$ \\
\hline \multirow[t]{2}{*}{ Abd. IV central mac } & B5 & + & - & + & + & + \\
\hline & B6 & $+/-$ & + & + & $+/-$ & - \\
\hline \multicolumn{2}{|c|}{ Trochanteral organ chaetae } & $22-27$ & $?$ & \pm 23 & $?$ & $35-52$ \\
\hline \multirow{2}{*}{\multicolumn{2}{|c|}{$\begin{array}{l}\text { Tibiotarsus III inner } \\
\text { smooth chaetae } \\
\text { Tenent hair }\end{array}$}} & 5 & - & + & + & 6 \\
\hline & & acuminate/capitate & acuminate/capitate & capitate & acuminate/capitate & acuminate \\
\hline Unguis teeth & ratio & b.t. $=$ m.t. $>$ a.t. & b.t. $=$ m.t. & b.t. $>$ m.t.. & b.t. $>$ m.t.. & b.t. $>$ m.t. $>$ a.t. \\
\hline Unguiculus lamellae & $\begin{array}{l}\text { a.t. } \\
\text { ai } \\
\text { pe }\end{array}$ & $\begin{array}{c}+ \\
\text { wide } \\
1 \text { tooth }\end{array}$ & $\begin{array}{c}- \\
\text { normal } \\
1 \text { tooth }\end{array}$ & $\begin{array}{c}- \\
\text { normal } \\
\text { smooth }\end{array}$ & $\begin{array}{c}- \\
\text { normal } \\
1 \text { tooth }\end{array}$ & $\begin{array}{c}+/- \\
\text { normal } \\
1 \text { tooth }\end{array}$ \\
\hline Manubrium dorsal & & $3+3$ & - & $4+4$ & $?$ & $4+4$ \\
\hline Dental spines & $\begin{array}{l}\text { number } \\
\text { distribution }\end{array}$ & $\begin{array}{c}16-20 \\
2-3 \text { rows grouped }\end{array}$ & $\begin{array}{c}? \\
\text { variable }\end{array}$ & $\begin{array}{c}20 \\
2 \text { rows grouped }\end{array}$ & $\begin{array}{c}16-42 \\
2-5 \text { rows }\end{array}$ & $\begin{array}{c} \pm 16 \\
2 \text { rows grouped }\end{array}$ \\
\hline
\end{tabular}

Legends: [] references; (+) present; (-) absent; ( \pm ) approximately; (/) or; (?) unknown/unclear; (S) smooth; (C) ciliate; (b.c.) basal chaetae; ungual teeth = (b.t.) basal paired teeth (m.t.) medial unpaired tooth, (a.p.) apical unpaired tooth; unguiculus lamellae = (ai) antero-internal lamella, (pe) postero-external lamella; $\left(^{*}\right)$ extra chaetae only on labial basomedian field;

$(* *)$ Soto-Adames and Anderson [48] only described the background colour, devoid of blue pigments. Distal antennae colour unknown for this species. 
Remarks: Dicranocentrus abestado sp. nov. belongs to the marias group of species as its dorsal head chaetotaxy is devoid of A1, S2 and all posterior mac except Pa5 and rarely Pp5 [12,47]. The new species is most similar to other Neotropical taxa from marias group, especially D. antillensis Mari-Mutt, 1979 [12], D. icelosmarias Soto-Adames and Anderson, 2017 [48], D. marias Wray, 1953 [49] and D. paramoensis Mari-Mutt, 1983 [47] since they also share seven mac on dorsal head S row (S0-1, S3-6), labial papilla E lateral process (1.p.) short, not reaching the apex of the papilla, labial basomedian field (labial triangle) with M1 chaeta ciliate and $\mathbf{m} 2$ and e smooth, Th. II lacking p5 mac and Abd. I-III with 3, 2 and 2 central mac, respectively. However, the new species can be readily separated from all others due to its colour pattern, with bluish pigments on thorax laterally to coxae, Abd. VI, distal femurs distally and all tibiotarsi (otherwise in the other species) and by its wide leaf-shaped unguiculus (normal in the other species), with one posterior tooth (absent in D. icelosmarias) (Table 2). The new species also lacks Pp5 mac on dorsal head (present in D. paramoensis), labial basomedian field unscaled with $\mathbf{R}$ chaeta ciliate (with scales in D. antillensis, $\mathbf{R}$ chaeta smooth at least in D. icelosmarias and D. marias), 3 basal post-labial basal chaetae around the cephalic groove ( 2 in D. paramoensis), Th. II with $3 \mathbf{m} 4$ and $4 \mathbf{p}$ mac (2 and 5, respectively, in D. antillensis and D. paramoensis), Th. III with 3, 0, 3 mac on a, $\mathbf{m}$ and $\mathbf{p}$ rows, respectively $(4,1,3$ in $D$. antillensis and 2, 1, 3 in D. paramoensis), unguis minute apical tooth (absent in D. antillensis, D. icelosmarias and D. marias) and dorsal manubrium with 3 smooth acuminate chaetae (absent in D. antillensis, 4 in D. icelosmarias and D. paramoensis). A detailed comparison of the main diagnostic features among such species is presented in Table 2.

Regarding the Brazilian species of Dicranocentrus, D. abestado sp. nov. is most similar to D. amazonicus, also from the marias group (Table 3). These species share similar colour patterns and dorsal head macrochaetotaxy, maxillary outer lobe basal chaeta acuminate, basomedian labial field unscaled with M1 chaeta ciliate, Th. II $\mathbf{p}$ row with four mac and Th. III a, $\mathbf{m}, \mathbf{p}$ rows with 3, 0, 3 mac, respectively. However, the new species differs from $D$. amazonicus especially by labial chaeta $\mathbf{R}$ ciliate (smooth in D. amazonicus), Abd. II with 2 central mac ( 1 in D. amazonicus), Abd. III with 1 lateral mac (2 in D. amazonicus), Abd. IV with 4-5 central mac ( 3 in D. amazonicus), unguiculus wide with one posterior tooth (normal and toothless in D. amazonicus) and unguis with a minute apical tooth (absent in D. amazonicus). Dicranocentrus pikachu is the second previously known species of the marias group from Brazil; however, it is remarkably different from D. abestado sp. nov., especially as it has 17 dorsal head An mac (11-12 in the new species), maxillary outer lobe basal chaeta blunt (acuminate in the new species), Th. II with 5-6 p chaetae (4 in the new species), Th. III $\mathbf{a}, \mathbf{m}, \mathbf{p}$ rows with 5, 1, 3 mac, respectively (3, 0, 3 in the new species), Abd. I with 5 central mac (vs. 3), Abd. III with 2 lateral mac (vs. 1), unguis lacking the apical tooth (vs. present) and about 50 dental spines (vs. 16-20).

Among the Brazilian species, D. abestado sp. nov. is the only one with 3 mac on Abd. I (Table 3). Dicranocentrus heloisae typically has 5 mac on Abd. I [18]; however, it was reported in specimens also with 3 [21]. Here, we disregard such observations, since it is possible that specimens of $D$. heloisae with 3 mac on Abd. I represent another species, as the macrochaetatoxy of Abd. I is quite a stable feature in Dicranocentrus; or such specimens may represent juveniles. Even so, D. abestado sp. nov. also differs by head with $0-1$ posterior mac (marias group), while in D. heloisae there are 2 (gracilis group). In addition, $D$. heloisae has a very distinct colour pattern, plus large number of chaetae in trochanteral organ and dental spines, compared to the new species. Further comparisons between both species and among other Brazilian taxa are presented in Table 3 and in the identification key in the discussion.

Dicranocentrus abestado sp. nov. also resembles species from the Old World from sundanensis and gracilis groups with 3 mac on Abd. I, especially D. gemellus Mari-Mutt 1985 [50], D. indicus Bonet, 1930 [51], D. inermodentes (Uchida, 1944) [52], D. luzonensis Mari-Mutt, 1985 [50], D. nepalensis Mari-Mutt and Bhattacharjee, 1980 [53], D. solomonensis Mari-Mutt, 1979 [12] and D. wangi Ma and Chen, 2007 [54] also by Abd. II-III central macrochaetotaxy with 2 and 2 mac, respectively. However, the Old World species have a more complex macrochaetotaxy on posterior head, which dismiss them from the marias group (head chaetotaxy unknown to D. indicus), as well as: 6-10 mac on p row of Th. II (4 in the new species), 9-12 mac on central Th. III (6 in the new species) and dental spines absent (except in 
D. luzonensis and D. wangi-also present in the new species). A detailed comparison among such species is presented in Table 4. Compared to species from the Old World from the sundanensis and gracilis groups with Abd I-II with 3 and 2 mac, respectively, but with 1 central mac on Abd. III instead (D. assimilis Schött, 1927 [55], D. inermis Schött, 1927 [55], D. javanus Yoshii and Suhardjono, 1989 [56], D. liuae $\mathrm{Xu}$ and Zhang, 2014 [57], D. meruensis Wahlgren, 1908 [58] and D. simplex Yosii, 1959 [59]), the new species differs beyond dorsal head and central Abd. III macrochaetotaxy, by having Th. II with $3 \mathbf{~ m} 4$ ( 2 in the others except in D. assimilis) and $4 \mathbf{p}$ mac (6-9 in the others), Th. III with 6 central mac (7-11 in the others), unguis with a minute apical tooth (absent in the others) and 16-20 dental spines (present only in D. meruensis-about 40-46 spines; in D. simplex-5-8 spines). Such species are compared in detail in Table 5. The type localities of all Dicranocentrus species with 3 mac on Abd. I are shown in Figure 7. 
Table 3. Comparison among the Brazilian Dicranocentrus species.

\begin{tabular}{|c|c|c|c|c|c|c|c|c|c|c|c|c|}
\hline & $\begin{array}{c}\text { Species } \\
\text { [References] }\end{array}$ & $\begin{array}{c}\text { abestado } \\
\text { sp. nov. }\end{array}$ & $\begin{array}{c}\text { albicephalus } \\
\text { [21] }\end{array}$ & $\begin{array}{c}\text { amazonicus } \\
\text { [17] }\end{array}$ & $\begin{array}{c}\text { bicolor } \\
\text { [13] }\end{array}$ & $\begin{array}{c}\text { cuprum } \\
\text { [20] }\end{array}$ & $\begin{array}{c}\text { heloisae } \\
{[16,18]}\end{array}$ & $\begin{array}{c}\text { magnus } \\
\text { [21] }\end{array}$ & $\begin{array}{c}\text { marimutti } \\
\text { [21] }\end{array}$ & $\begin{array}{c}\text { melinus } \\
{[20]}\end{array}$ & $\begin{array}{l}\text { pikachu } \\
\text { [21] }\end{array}$ & $\begin{array}{c}\text { termitophilus } \\
\text { [13] }\end{array}$ \\
\hline & Type Locality & $\begin{array}{c}\text { Chapada } \\
\text { Diamantina, } \\
\text { Bahia }\end{array}$ & $\begin{array}{c}\text { Serra dos } \\
\text { Órgãos, Rio } \\
\text { de Janeiro }\end{array}$ & $\begin{array}{c}\text { Reserva } \\
\text { Ducke, } \\
\text { Amazonas }\end{array}$ & $\begin{array}{l}\text { Blumenau, } \\
\text { Santa } \\
\text { Catarina }\end{array}$ & $\begin{array}{c}\text { Serra da } \\
\text { Gandarela, } \\
\text { Minas } \\
\text { Gerais }\end{array}$ & $\begin{array}{l}\text { Floresta da } \\
\text { Tijuca, Rio } \\
\text { de Janeiro }\end{array}$ & $\begin{array}{c}\text { Serra do } \\
\text { Japi, São } \\
\text { Paulo }\end{array}$ & $\begin{array}{c}\text { Serra dos } \\
\text { Órgãos, Rio } \\
\text { de Janeiro }\end{array}$ & $\begin{array}{c}\text { Serra da } \\
\text { Gandarela, } \\
\text { Minas } \\
\text { Gerais } \\
\end{array}$ & $\begin{array}{c}\text { Serra do } \\
\text { Japi, São } \\
\text { Paulo }\end{array}$ & Minas Gerais \\
\hline Characteristics & Species Group & marias & gracilis & marias & ? & gracilis & gracilis & gracilis & gracilis & gracilis & marias & ? \\
\hline Body & Ant. & + & + & + & Ant. II-IV & partially & + & partially & Ant. II-IV & + & partially & mostly \\
\hline \multirow{4}{*}{$\begin{array}{l}\text { bluish/violetish } \\
\text { pigmentation on: }\end{array}$} & Head (- eyes) & anteriorly & anteriorly & weakly & - & + & + & partially & - & anteriorly & - & anteriorly \\
\hline & Th. (dorsally) & - & + & - & - & + & - & + & Th. III & - & - & partially \\
\hline & Abd. (dorsally) & Abd. VI & + & - & - & + & - & partially & + & - & - & partially \\
\hline & Legs & partially & partially & weakly & mostly & proximally & $-/+$ & mostly & tibiotarsi & + & tibiotarsi & $-?$ \\
\hline \multicolumn{2}{|c|}{ Ant. Ilb smooth mac } & 3 & $-?$ & $?$ & $?$ & $-?$ & $6 ?$ & $1 ?$ & $4 ?$ & $-?$ & $-?$ & $?$ \\
\hline Interocular chaetae & & 5 & 3 & 3 & ? & 3 & 3 & 3 & 4 & 4 & 3 & ? \\
\hline \multirow{3}{*}{ Dorsal head mac } & An row & $11-12$ & $14-15$ & \pm 11 & ? & 14 & 12 & 13 & 15 & 13 & 17 & ? \\
\hline & S6i & + & + & + & ? & + & + & + & + & - & + & ? \\
\hline & posterior & 1 & 5 & $1^{*}$ & ? & 2 & 2 & 4 & 4 & 5 & 1 & ? \\
\hline $\begin{array}{l}\text { Maxillary outer } \\
\text { lobe b.c. }\end{array}$ & & acuminate & blunt & acuminate & ? & acuminate & acuminate & acuminate & acuminate & acuminate & blunt & ? \\
\hline \multirow{4}{*}{$\begin{array}{l}\text { Labial basomedian } \\
\text { field }\end{array}$} & M1 & C & C & C & $?$ & $\mathrm{~s}$ & $\mathrm{C}$ & C & C & C & C & $?$ \\
\hline & $\mathbf{R}$ (smaller) & C & C & S & ? & S & $\mathrm{s}$ & s & C & S & $\mathrm{C}$ & $?$ \\
\hline & extra chaetae & $0-1 \mathrm{~S}, 2-3 \mathrm{C}$ & - & $2 \mathrm{~S}, 1 \mathrm{C}$ & ? & - & $1 \mathrm{~S}$ & $1 \mathrm{~S}$ & $1 C^{* * *}$ & $1 \mathrm{~S}, 1 \mathrm{C}$ & $1 \mathrm{C}$ & ? \\
\hline & scales & - & - & - & ? & - & + & - & - & - & - & ? \\
\hline Th. II mac & p row & 4 & 6 & 4 & ? & 6 & 7 & 7 & 6 & 6 & $5-6$ & ? \\
\hline Th. III central mac & $\mathrm{a}, \mathrm{m}, \mathrm{p}$ rows & $3,0,3$ & $5,1,3$ & $3,0,3$ & ? & $5,1,3$ & $5,1,3$ & $5,1,3$ & $5,1,3$ & $5,1,3$ & $5,1,3$ & ? \\
\hline Abd. I mac & & 3 & 5 & 2 & ? & 5 & 5 & 5 & 5 & 5 & 5 & ? \\
\hline Abd. II mac & central & 2 & 2 & 1 & ? & 2 & 2 & 2 & 2 & 2 & 2 & ? \\
\hline Abd. III mac & lateral & 1 & 1 & 2 & ? & 2 & 2 & 2 & 2 & 2 & 2 & ? \\
\hline \multicolumn{2}{|c|}{$\begin{array}{l}\text { Abd. IV central } \\
\text { mac }\end{array}$} & $4-5$ & 5 & 3 & $?$ & 5 & 5 & 5 & 5 & 5 & 5 & $?$ \\
\hline \multicolumn{2}{|c|}{ Trochanteral organ chaetae } & $22-27$ & \pm 40 & \pm 30 & $?$ & \pm 70 & \pm 100 & \pm 100 & \pm 60 & \pm 65 & \pm 80 & $?$ \\
\hline \multicolumn{2}{|c|}{ Tenent hair } & $\begin{array}{l}\text { acuminate } \\
\text { /capitate }\end{array}$ & acuminate & capitate & capitate & acuminate & $\begin{array}{l}\text { acuminate/ } \\
\text { capitate }\end{array}$ & acuminate & acuminate & capitate & capitate & acuminate \\
\hline Unguis teeth & ratio & $\begin{array}{l}\text { b.t. }= \\
\text { m.t.>a.t. }\end{array}$ & b.t. $=$ m.t. & b.t. $=$ m.t. & b.t. $=$ m.t. & b.t. $=$ m.t. & $\begin{array}{l}\text { b.t. }= \\
\text { m.t.>a.t. }\end{array}$ & $\begin{array}{l}\text { b.t. }= \\
\text { m.t.>a.t. }\end{array}$ & $\begin{array}{l}\text { b.t. }=\text { m.t. }> \\
\text { a.t. }\end{array}$ & b.t. $=$ m.t. & b.t. $<$ m.t. & b.t. $=$ m.t. \\
\hline \multirow{3}{*}{$\begin{array}{l}\text { Unguiculus } \\
\text { lamellae }\end{array}$} & a.t. & + & - & - & - & - & + & + & + & - & - & - \\
\hline & ai & wide & normal & normal & normal & normal & normal & normal & narrow & normal & narrow & normal \\
\hline & pe & 1 tooth & 1 tooth & $\mathrm{s}$ & 1 tooth & 1 tooth & 1 tooth & 1 tooth & 1 tooth & 1 tooth ** & 1 tooth & $\mathrm{S}$ \\
\hline
\end{tabular}


Table 3. Cont

\begin{tabular}{|c|c|c|c|c|c|c|c|c|c|c|c|c|}
\hline & $\begin{array}{c}\text { Species } \\
\text { [References] }\end{array}$ & $\begin{array}{l}\text { abestado } \\
\text { sp. nov. }\end{array}$ & $\begin{array}{l}\text { albicephalus } \\
\text { [21] }\end{array}$ & $\begin{array}{c}\text { amazonicus } \\
\text { [17] }\end{array}$ & $\begin{array}{c}\text { bicolor } \\
\text { [13] }\end{array}$ & $\begin{array}{c}\text { cuprum } \\
\text { [20] }\end{array}$ & $\begin{array}{c}\text { heloisae } \\
{[16,18]}\end{array}$ & $\begin{array}{c}\text { magnus } \\
\text { [21] }\end{array}$ & $\begin{array}{c}\text { marimutti } \\
\text { [21] }\end{array}$ & $\begin{array}{c}\text { melinus } \\
\text { [20] }\end{array}$ & $\begin{array}{c}\text { pikachu } \\
\text { [21] }\end{array}$ & $\begin{array}{c}\text { termitophilus } \\
\text { [13] }\end{array}$ \\
\hline & Type Locality & $\begin{array}{c}\text { Chapada } \\
\text { Diamantina, } \\
\text { Bahia }\end{array}$ & $\begin{array}{c}\text { Serra dos } \\
\text { Órgãos, Rio } \\
\text { de Janeiro }\end{array}$ & $\begin{array}{c}\text { Reserva } \\
\text { Ducke, } \\
\text { Amazonas }\end{array}$ & $\begin{array}{c}\text { Blumenau, } \\
\text { Santa } \\
\text { Catarina }\end{array}$ & $\begin{array}{c}\text { Serra da } \\
\text { Gandarela, } \\
\text { Minas } \\
\text { Gerais }\end{array}$ & $\begin{array}{l}\text { Floresta da } \\
\text { Tijuca, Rio } \\
\text { de Janeiro }\end{array}$ & $\begin{array}{c}\text { Serra do } \\
\text { Japi, São } \\
\text { Paulo }\end{array}$ & $\begin{array}{c}\text { Serra dos } \\
\text { Órgãos, Rio } \\
\text { de Janeiro }\end{array}$ & $\begin{array}{c}\text { Serra da } \\
\text { Gandarela, } \\
\text { Minas } \\
\text { Gerais }\end{array}$ & $\begin{array}{c}\text { Serra do } \\
\text { Japi, São } \\
\text { Paulo }\end{array}$ & Minas Gerais \\
\hline $\begin{array}{l}\text { Characteristics } \\
\text { Dental spines }\end{array}$ & $\begin{array}{l}\text { Species Group } \\
\text { number } \\
\text { distribution }\end{array}$ & $\begin{array}{c}\text { marias } \\
16-20 \\
2-3 \text { rows } \\
\text { grouped }\end{array}$ & $\begin{array}{c}\text { gracilis } \\
\quad \pm 50 \\
\text { 5-6 rows } \\
\text { grouped }\end{array}$ & $\begin{array}{c}\text { marias } \\
\pm 19 \\
2 \text { rows } \\
\text { grouped }\end{array}$ & $\begin{array}{c}? \\
\pm 30 ? \\
4 \text { rows } \\
\text { grouped }\end{array}$ & $\begin{array}{l}\text { gracilis } \\
\quad \pm 50 \\
\text { grouped }\end{array}$ & $\begin{array}{l}\text { gracilis } \\
\quad \pm 60 \\
7 \text { rows }\end{array}$ & $\begin{array}{l}\text { gracilis } \\
\quad \pm 60 \\
6-7 \text { rows }\end{array}$ & $\begin{array}{c}\text { gracilis } \\
\pm 110 \\
\text { variable }\end{array}$ & $\begin{array}{l}\text { gracilis } \\
\pm 55 \\
\text { mostly } \\
\text { grouped }\end{array}$ & $\begin{array}{l}\text { marias } \\
\quad \pm 50 \\
\text { grouped }\end{array}$ & $\begin{array}{c}? \\
20-30 \\
3 \text { rows grouped }\end{array}$ \\
\hline
\end{tabular}

Legends: [] references; (+) present; (-) absent; ( \pm ) approximately; (/) or; (?) unknown/unclear; (S) smooth; (C) ciliate; (b.c.) basal chaetae; ungual teeth = (b.t.) basal paired teeth, (m.t.)

medial unpaired tooth, (a.p.) apical unpaired tooth; unguiculus lamellae $=\left(\right.$ ai) antero-internal lamella, (pe) postero-external lamella; $\left(^{*}\right)$ not represented in Bellini et al. [17]; $\left(^{* *}\right)$ represented on antero-external lamella lamella; $\left(^{* * *}\right)$ a1 not represented [21, pg 30, figure 15i]. D. silvestrii Absolon, 1903 was not included, see the discussion topic. 


\section{Discussion}

\subsection{Brazilian Species of Dicranocentrus}

The knowledge on Brazilian Dicranocentrus further increased during the past decade but is still limited at some level. With the description of $D$. abestado sp. nov. there are now 12 species of the genus recorded from Brazil (Table 1). Most of these species are only known from their type locality, with the exception of D. albicephalus (from Rio de Janeiro and Espírito Santo states), D. heloisae (from Rio de Janeiro, Espírito Santo, São Paulo, Minas Gerais and Bahia states) and D. silvestrii (Rio de Janeiro, Bahia and possibly Santa Catarina states) [4,19-21,61-63]. Of such species only D. silvestrii was recorded in other countries and it is considered widespread in South America, known from Argentina, Bolivia, Brazil, Chile, Peru and Venezuela [9], although its records are doubtful (see comments on this species, below). There are also few records of unidentified morphospecies from Brazil, as in [62,64], which combined to the nominal species records suggest that Dicranoncentrus is widely distributed at least in the Atlantic and Amazon Forest domains, as well as humid forested areas within the Caatinga domain.

Concerning the morphology of Brazilian species, D. albicephalus, D. cuprum, D. heloisae, D. magnus, D. marimutti, D. melinus and D. pikachu were very well studied and described/redescribed by Xisto and Mendonça [18,20,21]. Very few diagnostic features are unknown to these species, such as the number of smooth chaetae on furca and trunk sens formula, for example. Unfortunately, the type/analysed material of such species was lost in the fire in the National Museum of Rio de Janeiro at the end of 2018. The description of Dicranocentrus amazonicus is succinct as to most species of the genus, and some diagnostic features are unknown or unclear to it, such as the presence and distribution of smooth chaetae on antennae, tibiotarsi and furca, trunk sens formula, antennal and ventral head chaetotaxy (except labial and maxillary outer lobe chaetotaxy), number of tenaculum chaetae, as well as other features. The labral papillae shape and position and absence of scales on ventral tube are also unlikely characters and should be better investigated in this species [17]. Nevertheless, there are enough data to separate this species from other Brazilian and Neotropical taxa (Tables 2 and 3). Dicranocentrus bicolor, D. termitophilus and D. silvestrii, however, are poorly described and their names cannot be used with confidence for now, as already stated by Mari-Mutt [12] and Xisto and Mendonça [21]. Because of this we considered the three species as species inquirendae.

Dicranocentrus bicolor was collected in the Blumenau municipality, Santa Catarina state, inside nests of Eutermes arenarius termites [13]. Even though the type material of this species is in poor condition [12], there are sufficient data on the type locality and morphology, such as colour pattern, empodial complex and proximal dens-See Table 3 and Handschin ([13], pp. 23-24)—which allow for the collection of fresh samples, recognition of the species and its redescription. Dicranocentrus termitophilus-type material is also in poor condition [12], but the two type specimens were collected from Minas Gerais state-wrongly spelled "Minas Gueras" by Handschin [13]—inside nests of Cornitermes similis termites. Minas Gerais is the fourth largest state in Brazil with an area of about 586,500 square kilometres and encompass three very distinct biomes: Atlantic Forest (a tropical rainforest); Caatinga (a semi-arid landscape covered by a mosaic of very different phytophysiognomies) and Cerrado (a savanna-like domain). However, the few details on D. termitophilus morphology—see Table 3 and Handschin ([13], pp. 25-26) - the overall affinity of Brazilian Dicranocentrus species for humid forested areas (Table 1) and the biological association, even if accidental, with Cornitermes similis may provide enough data for the collection of new samples and the redescription of D. termitophilus. The very brief and generic description of D. silvestrii by Absolon ([24], pp. 105-106) makes it impossible to clearly separate this species from most taxa, and so we excluded it from Table 3. For instance, the body colour is described as yellowish, but also as dark in one species variation, without further details. Absolon's description also reports a capitate tenent hair (seen in several species of the genus and in a few polymorphic ones, as shown in Tables 2-5) and unguiculus with a medial tooth (also seen in several species, as shown in Tables 2-5). However, the most obscure data for this species is its type locality, which is listed only as 
"in South America" ([24], p. 106). Since its type material is apparently lost [12], the real type locality is unknown and none of the posterior descriptions consulted the type material $[65,66]$, the name $D$. silvestrii is highly dubious and should not be used in any case. One last detail about this species is the record of Börner [4] to D. silvestrii on orchids from "São Francisco", Brazil. This locality is as dubious as the species name, since "São Francisco" could represent several different localities far apart in Brazil. Further details on this issue are discussed in ([67], pp. 161). 
Table 4. Comparison among Dicranocentrus species from the Old Word with Abd. I-III with 3, 2 and 2 central mac, respectively.

\begin{tabular}{|c|c|c|c|c|c|c|c|c|}
\hline \multirow[b]{5}{*}{ Characteristics } & \multirow{2}{*}{$\begin{array}{c}\text { Species } \\
\text { [References] }\end{array}$} & gemellus & indicus & inermodentes & luzonensis & nepalensis & solomonensis & wang $i$ \\
\hline & & [50] & {$[12,51,54,60]$} & {$[12,44,52]$} & [50] & [53] & {$[12,44]$} & [54] \\
\hline & \multirow{2}{*}{ Type Locality } & Finschhafen, & Bandra, & Saipan, & Baguio, & Chainpur, & Guadalcanal, & Guangzhou, \\
\hline & & Papua New Guinea & India & Northern Mariana Is. & Philippine Is. & Nepal & Solomon Is. & China \\
\hline & Species Group & sundanensis & sundanensis? & sundanensis & sundanensis & gracilis & sundanensis & sundanensis \\
\hline \multicolumn{2}{|c|}{ Body bluish/violetish } & entire body or just & Ant., lateral Th. II & Ant., anterior head & Ant. and legs & Ant. and & $\begin{array}{l}\text { entire body, } \\
\text { except }\end{array}$ & Ant., distal \\
\hline \multicolumn{2}{|c|}{ pigmentation on } & antennae and legs & $\begin{array}{l}\text { and posterior } \\
\text { trunk segments } \\
\text { segments }\end{array}$ & and legs & (except femurs) & anterior head & $\begin{array}{l}\text { dorsal head and } \\
\text { furcula }\end{array}$ & $\begin{array}{c}\text { femurs and } \\
\text { tibiotarsi }\end{array}$ \\
\hline Interocular chaetae & & $4-5$ & $?$ & ? & 3 & ? & $?$ & $?$ \\
\hline \multirow[t]{5}{*}{ Dorsal head mac } & S2 & + & ? & + & + & - & + & + \\
\hline & Pa1 & + & ? & + & - & + & + & - \\
\hline & $\mathrm{Pa} 2$ & + & ? & + & + & + & + & + \\
\hline & Pp3 & + & ? & + & - & + & + & + \\
\hline & Pp5 & + & ? & + & + & - & + & + \\
\hline \multirow[t]{2}{*}{ Labral papillae } & inner & hooked & ? & conical & conical & conical & conical & conical \\
\hline & outer & hooked & ? & conical & rounded & conical & conical & conical \\
\hline \multirow{4}{*}{ Labial basomedian field } & M1 & S & ? & C & C & C & S & C \\
\hline & M2 & $\mathrm{s}$ & ? & $\mathrm{C}$ & $\mathrm{s}$ & $\mathrm{C}$ & $\mathrm{s}$ & $\mathrm{s}$ \\
\hline & $\mathbf{R}$ & $\mathrm{s}$ & ? & $\mathrm{S} / \mathrm{C}$ & S & $\mathrm{C}$ & S & S \\
\hline & extra chaetae & $2-3 \mathrm{~S}$ & ? & $0-1 \mathrm{~S}, 3-4 \mathrm{C}$ & $0-2 \mathrm{~S}, 1-2 \mathrm{C}$ & $2 \mathrm{C}$ & $4-6 \mathrm{~S}$ & $0-1 \mathrm{~S}, 2 \mathrm{C}$ \\
\hline \multirow[t]{2}{*}{ Th. II mac } & p1-3 group & 8 & 8 & 7 & 8 & 6 & 8 & $7-9$ \\
\hline & p5 mac & . & & + & - & - & & + \\
\hline Th. III central mac & $\mathrm{a}, \mathrm{m}, \mathrm{p}$ rows & $5,1,3$ & $5,1,3$ & $5,1,3$ & $5,1,3$ & $5,1,3$ & $5,1,5$ & $5,2,5$ \\
\hline \multicolumn{2}{|c|}{ Abd. IV central mac } & $4-5$ & $?$ & 3 & 3 & 4 & $4-5$ & $4-5$ \\
\hline \multicolumn{2}{|c|}{ Trochanteral organ chaetae } & 30-36 & 29 & ? & $24-37$ & ? & $?$ & $25-41$ \\
\hline Tibiotarsus III & inner & $+($ several) & - & - & $15-20$ & - & $+?$ & $0-2$ \\
\hline smooth chaetae & outer & + (several) & - & - & $1-2$ & - & $+?$ & 5-11 \\
\hline Tenent hair & & capitate/acuminate & capitate & capitate & acuminate & capitate & acuminate & acuminate \\
\hline Unguis teeth & $\begin{array}{c}\text { ratio } \\
\text { a.t. }\end{array}$ & $\begin{aligned} \text { b.t. }= & \text { m.t. }>\text { a.t. } \\
& +/-\end{aligned}$ & $\begin{aligned} \text { b.t. } & >\text { m.t. } \\
& -\end{aligned}$ & $\begin{aligned} \text { b.t. } & =\text { m.t. } \\
& -\end{aligned}$ & $\begin{array}{c}\text { b.t. }>\text { m.t. }=\text { a.t. } \\
+/-\end{array}$ & $\begin{array}{c}\text { b.t. }>\text { m.t. }=\text { a.t. } \\
+\end{array}$ & $\begin{array}{c}\text { b.t. }=\text { m.t. } \\
-\end{array}$ & $\begin{array}{c}\text { b.t. }>\text { m.t. } \\
-\end{array}$ \\
\hline \multirow{2}{*}{$\begin{array}{c}\text { Unguiculus } \\
\text { Tenaculum chaetae } \\
\text { chaetae }\end{array}$} & pe & $0-1$ tooth & smooth & smooth & 1 tooth & 1 tooth & 1 tooth & $0-1$ tooth \\
\hline & & $3-4$ & 1 & $?$ & $5-7$ & $?$ & $?$ & 4-10 \\
\hline \multirow{2}{*}{$\begin{array}{l}\text { Manubrium dorsal } \\
\text { smooth chaeta }\end{array}$} & number & 3-4 & - & - & 5-6 & - & 5 & $23-25$ \\
\hline & distribution & 2 rows & - & - & 2 rows & - & 2 rows & 3 rows \\
\hline \multicolumn{2}{|c|}{ Dens (basal) smooth chaeta } & 1 & ? & - & $1-3$ & - & 1 & $1-4$ \\
\hline \multicolumn{2}{|c|}{ Dens (dorsal) blunt mac } & - & + & - & - & - & - & - \\
\hline & number & - & - & - & $14-20$ & - & - & $8-13$ \\
\hline Dental spines & distribution & - & - & - & 1 row & - & - & 1 row \\
\hline
\end{tabular}

Legends: [] references; (+) present; (-) absent; (/) or; (?) unknown/unclear; (S) smooth; (C) ciliate; ungual teeth = (b.t.) basal paired teeth, (m.t.) medial unpaired tooth, (a.p.) apical unpaired tooth; unguiculus lamellae $=($ pe) postero-external lamella. 
Table 5. Comparison among Dicranocentrus species from the Old Word with Abd I-III with 3, 2 and 1 central mac, respectively.

\begin{tabular}{|c|c|c|c|c|c|c|c|}
\hline \multirow[b]{5}{*}{ Characteristics } & \multirow{2}{*}{$\begin{array}{c}\text { Species } \\
\text { [references] }\end{array}$} & assimilis & inermis & javanus & liuae & meruensis & simplex \\
\hline & & {$[12,44,55]$} & {$[12,55]$} & [56] & [57] & {$[12,44,58]$} & {$[56,59]$} \\
\hline & \multirow{2}{*}{ Type Locality } & Debundscha, & Debundscha, & Bogor, West & Shitai, & Meru, & Bukit Timah, \\
\hline & & Cameroon & Cameroon & Java, Indonesia & China & Kenya & Singapore \\
\hline & Species Group & gracilis & gracilis & sundanensis & sundanensis & gracilis & sundanensis \\
\hline \multicolumn{2}{|c|}{ pigmentation on } & $\begin{array}{l}\text { Entire body, except } \\
\text { dorsal head and } \\
\text { trunk } \\
\text { and ventral dens }\end{array}$ & $\begin{array}{l}\text { Ant., legs and } \\
\text { Abd. V-VI }\end{array}$ & Ant. only & $\begin{array}{l}\text { Ant., femurs and } \\
\text { tibiotarsi }\end{array}$ & $\begin{array}{l}\text { Entire body or } \\
\text { just Ant. and legs }\end{array}$ & $\begin{array}{l}\text { Ant., legs and } \\
\text { ventral body }\end{array}$ \\
\hline \multirow[t]{4}{*}{ Dorsal head mac } & S2 & - & - & + & + & - & + \\
\hline & Pa1 & - & - & + & + & - & - \\
\hline & Рp3 & + & - & + & + & + & + \\
\hline & Pp5 & + & - & + & + & - & + \\
\hline \multirow{4}{*}{$\begin{array}{l}\text { Labial basomedian } \\
\quad \text { field }\end{array}$} & M1 & $?$ & ? & $\mathrm{S}$ & $\mathrm{C}$ & $\mathrm{C}$ & ? \\
\hline & M2 & ? & ? & $\mathrm{S}$ & $\mathrm{S}$ & $\mathrm{S}$ & $?$ \\
\hline & $\mathbf{R}$ & ? & ? & $\mathrm{C}$ & $\mathrm{S}$ & $\mathrm{S}$ & ? \\
\hline & extra chaetae & ? & ? & $3 C$ & $4 \mathrm{~S}$ & $6 \mathrm{C}$ & ? \\
\hline \multirow[t]{3}{*}{ Th. II mac } & m4 group & 3 & 2 & 2 & 2 & 2 & 2 \\
\hline & p1-3 group & 6 & 7 & 6 & 8 & 6 & $6-7$ \\
\hline & p5 mac & - & - & + & + & - & - \\
\hline Th. III central mac & $\mathrm{a}, \mathrm{m}, \mathrm{p}$ rows & $4,2,3$ & $4,2,3$ & $5,1,3$ & $5,1,3-4$ & $4,2,3$ & $4,0,3$ \\
\hline \multicolumn{2}{|c|}{ Trochanteral organ chaetae } & $?$ & $?$ & 50 & 30 & $?$ & 70 \\
\hline Tibiotarsus III & inner & $+?$ & $+?$ & - & $+?$ & $+?$ & $?$ \\
\hline smooth chaetae & outer & - & $+?$ & - & $-?$ & - & $?$ \\
\hline Tenent hair & & acuminate & capitate & acuminate & acuminate & acuminate & acuminate \\
\hline Unguis teeth & $\begin{array}{l}\text { ratio } \\
\text { m.t. }\end{array}$ & $\begin{aligned} \text { b.t. } & >\text { m.t. } \\
& +\end{aligned}$ & $\begin{aligned} \text { b.t. } & =\text { m.t. } \\
& +\end{aligned}$ & $\begin{aligned} \text { b.t. } & >\text { m.t. } \\
& +\end{aligned}$ & $\begin{aligned} \text { b.t. } & =\text { m.t. } \\
& +\end{aligned}$ & $\begin{aligned} \text { b.t. } & =\text { m.t. } \\
& +\end{aligned}$ & $\begin{array}{l}\text { b.t. only * } \\
-?\end{array}$ \\
\hline \multirow[t]{2}{*}{$\begin{array}{l}\text { Unguiculus } \\
\text { lamellae }\end{array}$} & ai & acuminate & truncate, 1 tooth & acuminate & acuminate & acuminate & acuminate \\
\hline & pe & 1 tooth & 1 tooth & smooth & smooth & 1 tooth & smooth \\
\hline \multicolumn{2}{|l|}{ Tenaculum chaetae } & $?$ & $?$ & 2 & $2-4$ & $?$ & $?$ \\
\hline \multirow{2}{*}{$\begin{array}{l}\text { Manubrium dorsal } \\
\text { smooth chaeta }\end{array}$} & number & ? & ? & 6 & $+?$ & $+?$ & 3 \\
\hline & distribution & ? & ? & 2 rows & $+?$ & $+?$ & 2 rows \\
\hline \multicolumn{2}{|c|}{ Dens (basal) smooth chaeta } & ? & ? & 1 & 5 & $+?$ & 2 \\
\hline \multicolumn{2}{|c|}{ Dens (dorsal) blunt mac } & - & - & + & - & - & - \\
\hline Dental spines & number & - & - & - & - & $\pm 40-46$ & $5-8$ \\
\hline Dental spines & distribution & - & - & - & - & $3-4$ rows & 1 row \\
\hline
\end{tabular}

Legends: [] references; (+) present; (-) absent; ( \pm ) approximately; (?) unknown/unclear; (S) smooth; (C) ciliate; ungual teeth = (b.t.) basal paired teeth, (m.t.) medial unpaired tooth; unguiculus lamellae $=\left(\right.$ ai) antero-internal lamella, (pe) postero-external lamella. ${ }^{*}$ Yosii's drawing [59, pg. 41, figure 24D] and his text description point to the presence of only the medial tooth, however it is more likely it is the basal pair of minute teeth more distally displaced. 
A key to the Brazilian species of Dicranocentrus is presented below.

Identification key of Brazilian Dicranocentrus species *,**

1. Head post-occipital series with $0-1$ (Pa5) mac (Figure 2F) ... (marias group) 2

- Head post-occipital series with 2 or more mac ... (gracilis group) 4

2. Th. II with 5-6 posterior mac; Th. III with 9 central mac; Abd. I with 5 mac ... D. pikachu Xisto and Mendonça, 2017

- Th. II with 4 posterior mac; Th. III with 6 central mac; Abd. I with 3 or less mac (Figure 4A-C) ... 3

3. Abd. I-II with 3 and 2 central mac, respectively (Figure 4C-D); Abd. III with 1 lateral mac; Abd. IV with 4-5 central mac (Figure 5A-B); unguiculi leaf shaped and wide, with one posterior tooth (Figure 6B) ... D. abestado sp. nov.

- Abd. I-II with 2 and 1 central mac, respectively; Abd. III with 2 lateral mac; Abd. IV with 3 central mac; unguiculi normal without posterior teeth ... D. amazonicus Bellini, Moraes and Oliveira, 2013

4. Head post-occipital series with 2 mac ... 5

- Head post-occipital series with $4-5$ mac ... 6

5. Trunk pigmented; head An row with 14 mac; labial basomedian field with $\mathbf{m} \mathbf{1}$ smooth and devoid of extra chaetae and scales; Th. II with 6 posterior mac; unguis apical tooth absent ... D. cuprum Xisto and Mendonça, 2016

- Trunk depigmented; head An row with 12 mac; labial basomedian field with M1 ciliate, 1 extra smooth chaeta and scales present; Th. II with 7 posterior mac; unguis apical tooth present ... D. heloisae Arlé and Mendonça, 1982

6. Head post-occipital series with 4 mac; unguis apical tooth present ... 7

- Head post-occipital series with 5 mac; unguis apical tooth absent ... 8

7. Th. III to Abd. V and tibiotarsi distally pigmented; head An row with 15 mac; labial basomedian field with $\mathbf{R}$ and 1 extra chaeta ciliate; Th. II with 6 posterior mac; trochanteral organ with about 60 spine-like chaetae; dens with about 110 spines ... D. marimutti Xisto and Mendonça, 2017

- Head, trunk and coxae to most of tibiotarsi pigmented; head An row with 13 mac; labial basomedian field with $\mathbf{r}$ and 1 extra chaeta smooth; Th. II with 7 posterior mac; trochanteral organ with about 100 spine-like chaetae; dens with about 60 spines ... D. magnus Xisto and Mendonça, 2017

8. Trunk and manubrium heavily pigmented; head with S6i mac and 3 interocular chaetae; maxillary outer lobe basal chaeta blunt; labial basomedian field with $\mathbf{R}$ ciliate and devoid of extra chaetae; Abd. III with 1 lateral mac ... D. albicephalus Xisto and Mendonça, 2017

- Pigments restricted to antennae and legs (weakly); head devoid of S6i mac, with 4 interocular chaetae; maxillary outer lobe basal chaeta acuminate; labial basomedian field with $\mathbf{r}$ smooth and 2 extra chaeta (one smooth, the other ciliate); Abd. III with 2 lateral mac ... D. melinus Xisto and Mendonça, 2016

Notes: $\left({ }^{*}\right)$ D. bicolor Handschin, 1924, D. termitophilus, Handschin 1924 and D. silvestrii Absolon, 1903 were not included since they were considered species inquirendae (see the previous topic); $\left.{ }^{* *}\right)$ All Brazilian species of Dicranocentrus are only known from Brazil, with the exception of D. silvestrii.

\subsection{Notes on Dicranoncentrus Chaetotaxy}

Dicranocentrus species morphology is widely variable as partially represented in Tables 2-5. Dorsal head macrochaetotaxy can have, among other chaetae, S2, S6i and several posterior mac (on Pa, $\mathbf{P m}$ and Pp rows) or can be devoid of at least some of these mac [12]. Trunk dorsal macrochaetotaxy of most species has few or none secondary mac, other than those on the Th. II anterior collar, $\mathrm{m} 4$ and $\mathbf{p}$ groups, and $\mathbf{m} 3 \mathbf{e}$ on Abd. II, but differences in the presence and number of some primary mac or their multiples occur. Such data are of taxonomic relevance, especially on Th. II-III and Abd. I [12]. The number and displacement of smooth acuminate chaetae on proximal antennae, tibiotarsi, manubrium and proximal dens are of specific value, as well as the presence, number and position of dental spines. Labial basomedian field $\mathbf{M}$ and $\mathbf{R}$ chaetae multiples, which can include scales on some 
species, are so inconstant and can be so abundant that in some cases can prevent a clear understanding of which are some of the primary chaetae. Even the tenaculum corpus chaetotaxy diverges among the species (and can be polymorphic in few ones), as represented in Tables 4 and 5. Such variations among the species plus our finding of D. abestado sp. nov. with an unexpected number of seven sens on Abd. V (previously known as only four, but unknown to most taxa) may point out to an artificial status for Dicranocentrus. For instance, Sinodicranocentrus Zhang, 2020 [15] was recently erected based especially on ungual morphology and Abd. V S-chaetotaxy, and it was supported by molecular data; otherwise Dicranocentrus would be paraphyletic ([15], p. 15, Figure 1). In this sense it is possible that the widely variable morphology of Dicranocentrus may hide more distinct lineages of Heteromurini, and further studies on its morphology and systematics may unveil this condition. This approach could also confirm/refute Mari-Mutt's groups of species [12].

\subsection{Notes on Dicranocentrus Species Groups and Related Genera}

Mari-Mutt's groups of species based on dorsal head macrochaetatoxy are quite useful for the current taxonomy of Dicranocentrus [12,17,18,20,21,41,57]. Distribution endorses Mari-Mutt's groups at some level [12] (as partially represented in Figure 7). For instance, the marias group is almost exclusively Neotropical, with the exception of D. spinosus Prabhoo, 1971 [68] described from India. This group is well delimited by the reduction in dorsal head macrochaetotaxy, devoid of $\mathbf{S} 2$ and most post-occipital mac, aside from Pa5 (which can be rarely absent as well) and Pp5 (only seen in D. paramoensis) [12].

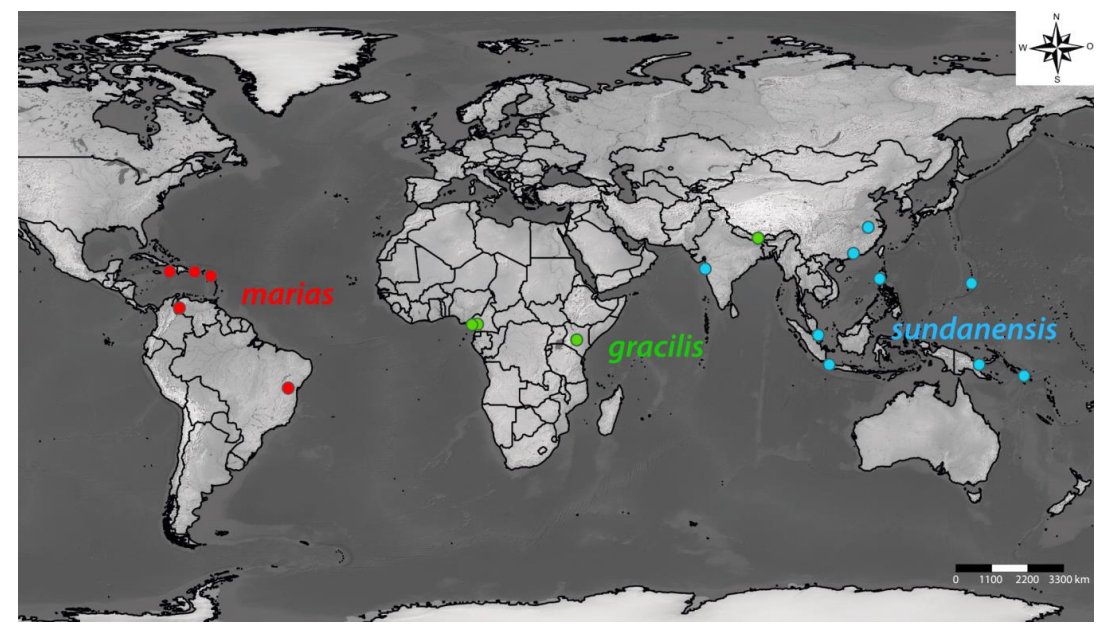

Figure 7. Type localities of all 18 Dicranocentrus species with 3 mac on Abd. I, distributed in the marias group (red dots, Neotropical region) and in the gracilis and sundanensis groups (green and blue dots, respectively, Paleotropical region). Further data on such species are presented in Tables 2, 4 and 5.

The gracilis and sundanensis groups are characterized by a more complex dorsal head macrochaetotaxy, with post-occipital series with two or more mac, but they differ from each other by the presence (sundanensis) or absence (gracilis) of $\mathbf{S 2}$ mac. Other morphological features of both are mixed and should be better investigated since they raise doubts if the gracilis and sundanensis groups are really independent taxa. For example, both groups hold species with one or two central mac on Abd. III, with or without dental spines, and with or without a very peculiar proximal blunt mac on dorsal dens, described to South Asian species as D. janetscheki Yosii, 1971 [69], D. indicus and D. javanus, from the sundanensis group, and D. fraternus Mari-Mutt and Bhattacharjee, 1980 [53], from the gracilis group $[53,56,60,69]$. The head chaetotaxy of D. janetscheki and D. indicus are unknown, and thus it is not entirely clear if they really belong to the sundanensis group [12,69]; D. javanus, however, belong to that group [56].

The presence of 1 proximal dental mac was also reported to Falcomurus chilikaensis Mandal, 2018 [70] (Heteromurini), the sole species of the genus described from India. This species was 
compared specially with Heteromurus due to the presence of only Ant. I subdivided (so, with five antennal segments) and Abd. I devoid of mac [70]. However, there are incongruences on this species description [15], which may point to an inaccurate interpretation of Abd. I chaetotaxy as well. Additionally, Dicranocentrus specimens can rarely have four or five antennal segments, as juveniles or when the antennae are regenerating [12]. Finally, among the Orchesellidae, only species of Dicranocentrus and now Falcomurus have a proximal dental mac. Because of this, it is possible that Falcomurus represents a Dicranocentrus species.

Mari-Mutt's phylogeny suggested the marias and gracilis groups had a close ancestral link [12]. Due to the wide Pantropical distribution of the gracilis group [3,12], it is possible it was already established at least during the Jurassic (about 180 mya), and the marias group emerged from a gracilis-like ancestor after the brake of western Gondwana in the Neotropical Region. In this sense, the presence of $D$. spinosus of the marias group in India was possibly due to: (1) human-mediated dispersal events, such as plant and soil carrying during the past five centuries; (2) it emerged independently from the Neotropical marias group, and its resemblance with other taxa is due to convergence. Mari-Mutt's phylogeny also suggested that the sundanensis group and Pseudodicranocentrus Mari-Mutt, 1981 [45] (formerly Dicranocentrus circulatus group) are closely related [12]. The distribution of the sundanensis group is Oriental to Australasian (as partially represented in Figure 7), while Pseudodicranocentrus is endemic to Mexico and Guatemala [3,9,45]. If Mari-Mutt's hypothesis is correct, the presence of Pseudodicranocentrus in Central America possibly occurred by the dispersal of its ancestor by the Pacific Ocean, similarly to the model of Christiansen and Bellinger for the Hawaiin colonization [71].

Below, we provide an identification key to all species of Dicranocentrus with 3 mac on Abd. I. They belong to marias, sundanensis and gracilis groups. Further data on such species are presented in the remarks on the new species and in Tables 2, 4 and 5.

Identification key of Dicranocentrus species with 3 mac on Abd. I

1. Dorsal dens with 1 proximal blunt mac ... 2

-. Dorsal dens devoid of blunt mac... 3

2. Th. II with 8 posterior mac, $\mathbf{p} 5$ mac absent; Abd. III with 2 central mac; trochanteral organ with about 29 spine-like chaetae; tenent hairs capitate; tenaculum with 1 chaeta; dorsal manubrium devoid of smooth chaetae ... D. indicus Bonet, $1930^{*}$

- Th. II with 7 posterior mac, p5 mac present; Abd. III with 1 central mac; trochanteral organ with about 50 spine-like chaetae; tenent hairs acuminate; tenaculum with 2 chaetae; dorsal manubrium with 6 smooth chaetae in 2 rows ... D. javanus Yoshii and Suhardjono, 1989

3. Head post-occipital series with 0-1 (Pa5) mac (Figure 2F); Neotropical distribution ... (marias group) 4

- Head post-occipital series with 2 or more mac; Old World distribution (Figure 7) . . 8

4. Labial basomedian field devoid of R chaeta; Th. III with 8 central mac; Abd. IV devoid of B5 mac ... D. antillensis Mari-Mutt, 1979

- Labial basomedian field with $\mathbf{R}$ chaeta; Th. III with 6 central mac; Abd. IV with $\mathbf{B} 5$ mac ... 5

5. Labial basomedian field with 6-7 extra ciliate chaetae; Th. II $\mathbf{m} 4$ group with 2 mac, with 5 posterior mac; Th. III a, m, p rows with 2, 1, 3 mac respectively ... D. paramoensis Mari-Mutt, 1983

- Labial basomedian field with 1-3 extra ciliate chaetae; Th. II $\mathbf{m} 4$ group with 3 mac, with 4 posterior mac; Th. III a, $\mathbf{m}, \mathbf{p}$ rows with $3,0,3$ mac respectively $\ldots 6$

6. Labial basomedian field $\mathbf{R}$ chaeta ciliate; ungues with apical tooth; unguiculi leaf shaped and wide ... D. abestado sp. nov.

- Labial basomedian field $\mathbf{r}$ chaeta smooth; ungues devoid of apical tooth; unguiculi normal ... 7

7. Labial basomedian field with 3-4 extra smooth chaetae; unguiculi devoid of outer teeth ... D. icelosmarias Soto-Adames and Anderson, 2017

- Labial basomedian field with 1-3 extra smooth chaetae; unguiculi with 1 outer tooth ... D. marias Wray, 1953

8. Head sutural series with 7 mac (S2 absent, Figure 2F) ... (gracilis group) 9 
- Head sutural series with 8 mac (S2 present) ... (sundanensis group) 12

9. Head post-occipital series with 10 mac; Th. III a, m, p series with 5, 1, 3 mac, respectively; Abd. III with 2 central mac (Figure 5A) ... D. nepalensis Mari-Mutt, 1980

- Head post-occipital series with 6 or less mac; Th. III a, m, p series with 4, 2, 3 mac, respectively; Abd. III with 1 central mac ... 10

10. Dental spines present... D. meruensis Wahlgren, 1908

- Dental spines absent ... 11

11. Head with Pp3 and Pp5 mac; Th. II $\mathbf{m} 4$ group with 3 mac, with 6 posterior mac; tenent hairs acuminate; unguiculi acuminate without inner teeth ... D. assimilis Schött, 1927

- Head devoid of Pp3 and Pp5 mac; Th. II $\mathbf{m} 4$ group with 2 mac, with 7 posterior mac; tenent hairs capitate; unguiculi truncate with 1 inner tooth ... D. inermis Schött, 1927

12. Abd. III with 1 central mac... 13

- Abd. III with 2 central mac (Figure 5A) ... 14

13. Head with Pa1 mac; Th. II with 9 posterior mac, p5 mac present; Th. III a, $\mathbf{m}, \mathbf{p}$ series with 5 , 1, 3-4 mac, respectively; trochanteral organ with about 30 spine-like chaetae; dental spines absent ... D. liuae $\mathrm{Xu}$ and Zhang, 2014

- Head devoid of Pa1 mac; Th. II with 6-7 posterior mac, p5 mac absent; Th. III a, m, p series with 4, 0, 3 mac, respectively; trochanteral organ with about 70 spine-like chaetae; dental spines present ... D. simplex Yosii, 1959

14. Dental spines present ... 15

- Dental spines absent ... 16

15. Dorsal head Pp3 mac absent; Th. II devoid of p5 mac; Th. III with 9 central mac; Abd. IV with 3 central mac; dorsal manubrium with 5-6 smooth chaetae; dens with 14-20 spines ... D. luzonensis Mari-Mutt, 1985

- Dorsal head Pp3 mac present; Th. II with p5 mac; Th. III with 12 central mac; Abd. IV with 4-5 central mac; dorsal manubrium with 23-25 smooth chaetae; dens with 8-13 spines ... D. wangi Ma and Chen, 2017

16. Labial basomedian field chaetae M1-2 ciliate; Th. II p1-3 group with 7 mac, p5 mac present; Abd. IV with 3 central mac; manubrium devoid of smooth chaetae ... D. inermodentes (Uchida, 1944)

- Labial basomedian field chaetae m1-2 smooth; Th. II p1-3 group with 8 mac, p5 mac absent; Abd. IV with 4-5 central mac; dorsal manubrium with 2 rows of smooth chaetae ... 17

17. Labral papillae hooked; labial basomedian field with 2-3 extra smooth chaetae; Th. III with 9 central mac; dorsal manubrium with 3-4 smooth chaetae on each row ... D. gemellus Mari-Mutt, 1985

- Labral papillae conical; labial basomedian field with 4-6 extra smooth chaetae; Th. III with 11 central mac; dorsal manubrium with 5 smooth chaetae on each row ... D. solomonensis Mari-Mutt, 1979

Notes: $\left({ }^{*}\right)$ The head chaetotaxy of D. indicus is unknown (Table 4). Nevertheless, Mari-Mutt [12] suggests it is from the sundanensis group, closely related to D. inermodentes. Ma and Chen [54] key considered the S2 mac present in D. indicus, possibly based on [12], but apparently no material was examined. Because of this we considered such information as dubious.

\subsection{Notes on Heteromurinae Systematics}

Although the internal evolution of Heteromurinae was, to some extent, recently studied [15], its affinities with other Entomobryoidea are not entirely clear [2,72]. Recent advances in Entomobryoidea/Entomobryidae systematics support both a closer relationship of Heteromurinae with derived taxa as the Entomobryinae, Seirinae, Lepidocyrtinae and Paronellidae [26,72-74], or with other Orchesellidae [2,75]. For now, Heteromurinae is grouped with unscaled Orchesellidae [2], but the morphology of this subfamily, with an overall reduction in trunk dorsal mac multiples, several primary chaetae as mic, the presence of coarsely ciliate scales as secondary coverage and reduced S-chaetotaxy formula (2, 2|1, 3, 3 on Th. II to Abd. III) suggest that this taxon is possibly more derived than most unscaled Orchesellidae [12,40,57,72]. Even though Heteromurinae and other Orchesellidae share a short 
Abd. IV and a postantennal organ (seen only in Alloscopus among the Heteromurinae, variable among other Orchesellidae), such features should be interpreted as plesiomorphies, as well as the presence of hook-like labral papillae and tenaculum with more than one chaeta, also seen in some of their species. In this context, if we consider the alternative hypothesis to Entomobryoidea topology as Orchesellidae + (Heteromurinae + Entomobryidae/Paronellidae), the presence of scales could be the synapomorphy of the more derived Entomobryoidea, including Heteromurinae, and it was secondarily lost possibly more than one time among the Entomobryinae $[75,76]$. To support this interpretation, the basal Entomobryinae and all Seirinae also share coarsely ciliate scales with the Heteromurinae [72,75,77].

\section{Conclusions}

After the description of Dicranocentrus abestado sp. nov. there are now 12 species of the genus recorded from Brazil. Its Abd. V S-chaetotaxy with seven sens, unmatched in the genus, combined with the widely variable morphology of Dicranocentrus species, and the recent description of Sinodicranocentrus based on ungual morphology and S-chaetotaxy raises doubts about the validity of the former genus. Regarding the Brazilian species inquirendae, there is arguably enough data for the collection of fresh specimens of D. bicolor and D. termitophilus and their redescription. Conversely, D. silvestrii identity, including its real type locality, remains obscure and such a name cannot be used with certainty. The Heteromurinae position within Entomobryoidea remains puzzling, but the morphology, especially the presence of scales, may indicate its affinities with more derived taxa than the unscaled Orchesellidae.

Author Contributions: Conceptualization, B.C.B.; methodology, O.J.R.S., B.C.B., and N.G.C.; software, O.J.R.S. and B.C.B.; validation, B.C.B. and N.G.C.; formal analysis, O.J.R.S. and B.C.B.; investigation, O.J.R.S. and B.C.B.; resources, B.C.B.; data curation, B.C.B., N.G.C., and O.J.R.S.; writing-original draft preparation, B.C.B. and O.J.R.S.; writing-review and editing, B.C.B. and N.G.C.; visualization, O.J.R.S., B.C.B., and N.G.C.; supervision, B.C.B.; project administration, B.C.B.; funding acquisition, B.C.B. All authors have read and agreed to the published version of the manuscript.

Funding: This research was supported by UFRN. B.C.B. was granted by CNPq and CAPES (PPBio Programa de Pesquisa em Biodiversidade-Invertebrados; PROTAX II, process \#156828/2016-01 and PQ2018, process \# 305426/2018-4).

Acknowledgments: We thank the anonymous referees for revising this manuscript.

Conflicts of Interest: The authors declare no conflict of interest.

\section{References}

1. Womersley, H. A preliminary account of the Collembola-Arthropleona of Australia II (Entomobryoidea). Trans. R. Soc. S. Aust. 1934, 58, 86-138.

2. Zhang, F.; Bellini, B.C.; Soto-Adames, F.N. New insights into the systematics of Entomobryoidea (Collembola: Entomobryomorpha): First instar chaetotaxy, homology and classification. Zool. Syst. 2019, 44, 249-278. [CrossRef]

3. Bellinger, P.F.; Christiansen, K.A.; Janssens, F. Checklist of the Collembola of the World. Available online: http://www.collembola.org (accessed on 31 August 2020).

4. Börner, C. Das System der Collembolen-nebst Beschreibung neuer Collembolen des Hamburger Naturhistorischen Museums. Mit. aus dem Nat. Mus. Hambg. 1906, 23, 147-188.

5. Schäffer, C. Die Collembolen der Umgebung von Hamburg und benachtbaren Gebiete. Mit. aus dem Nat. Mus. Hambg. 1896, 13, 147-216.

6. Zeppelini, D.; Queiroz, G.C.; Bellini, B.C. Collembola in Catálogo Taxonômico da Fauna do Brasil. PNUD. Available online: http://fauna.jbrj.gov.br/fauna/faunadobrasil/379/ (accessed on 31 August 2020).

7. Schött, H. Beiträge zur kenntnis der insektenfauna von Kamerun, Bihang till K. Sven. Vetenskapsakad. Handl. Stockh. 1893, 19, 5-28.

8. Templeton, R.; Westwood, J.O. Thysanurae Hibernicae, or descriptions of such species of spring-tailed insects (Podura and Lepisma, Linn.,) as have been observed in Ireland. Descriptions of the Irish Species of Thysanura. Trans. Entomol. Soc. Lond. 1836, 1, 92-98. [CrossRef]

9. Mari-Mutt, J.A.; Bellinger, P.F. A Catalog of Neotropical Collembola, Including Nearctic Areas of Mexico. In Flora and Fauna Handbook 5, 1st ed.; Sandhill Crane Press: Gainesville, FL, USA, 1990; pp. 1-237. 
10. Mari-Mutt, J.A.; Bellinger, P.F. Supplement to the catalog of the Neotropical Collembola. Caribb. J. Sci. 1996, 32, 166-175.

11. Mari-Mutt, J.A.; Bellinger, P.F.; Janssens, F. Checklist of the Collembola: Supplement to the Catalog of the Neotropical Collembola. Available online: http://www.collembola.org/publicat/neotrcat.htm (accessed on 31 August 2020).

12. Mari-Mutt, J.A. A revision of the genus Dicranocentrus Schött (Insecta: Collembola: Entomobryidae). Agric. Exp. Sta. Bull. 1979, 259, 1-79.

13. Handschin, E. Neue myrmecophile und termitophile Collembolenformen aus Süd Amerika. Neue Beitr. Syst. Insektenk. Berlin 1924, 3, 13-28.

14. Cipola, N.G.; Oliveira, F.G.L.; Morais, J.W.; Bellini, B.C. The Heteromurini Absolon \& Ksenemann (Collembola, Entomobryidae): A review of the genera status and diagnoses, keys for species of Alloscopus Börner and Heteromurtrella Mari-Mutt and description of a new species. Zootaxa 2016, 4084, 151-186. [CrossRef]

15. Zhang, F.; Cipola, N.G.; Pan, Z.-X.; Ding, Y. New insight into the systematics of Heteromurini (Collembola: Entomobryidae: Heteromurinae) with special reference to Alloscopus and Sinodicranocentrus gen. $\mathrm{n}$. Arth. Syst. Phylogeny 2020, 78, 1-16. [CrossRef]

16. Arlé, R.; Mendonça, C. Estudo preliminar das espécies de Dicranocentrus Schött, 1893, ocorrentes no Parque Nacional da Tijuca, Rio de Janeiro (Collembola). Rev. Brasil. Biol. 1982, 42, 41-49.

17. Bellini, B.C.; Morais, J.W.; Oliveira, F.G.L. A new species of Dicranocentrus Schött (Collembola, Entomobryidae, Orchesellinae) from Brazilian Amazon. Zootaxa 2013, 3709, 296-300. [CrossRef] [PubMed]

18. Xisto, T.; Mendonça, M.C. Redescription of Dicranocentrus heloisae Arlé \& Mendonça, 1982 (Collembola: Entomobryidae). J. Insect Biodivers. 2014, 2, 1-10. [CrossRef]

19. Xisto, T.; Mendonça, M.C.; Abrantes, E.A. Dicranocentrus heloisae Arlé \& Mendonça, 1982 (Collembola: Entomobryidae): First record of the genus in Espírito Santo state, Brazil. Check List 2015, 11, 1-3. [CrossRef]

20. Xisto, T.; Mendonça, M.C. Two new species of Dicranocentrus Schött, 1893 (Collembola: Entomobryidae) from Serra do Gandarela, Minas Gerais State, Brazil. Zootaxa 2016, 4079, 217-228. [CrossRef] [PubMed]

21. Xisto, T.; Mendonça, M.C. New species and new records of Dicranocentrus Schött, 1893 (Collembola: Entomobryidae) from Southeastern Brazil. Insect Syst. Evol. 2017, 49, 23-58. [CrossRef]

22. Absolon, K.; Ksenemann, M. Troglopedetini. Vergleichende Studie über eine altertümliche höhlenbewohnende Kollembolengruppe aus den dinarischen Karstgebieten. Stud. Geb. Karst. Höhlenkunde Eiszeitforsch. Nachbargeb. 1942, 16, 5-57.

23. Mari-Mutt, J.A. Heteromurtrella, a new tropical subgenus of Heteromurus with descriptions of two new species (Insecta: Collembola: Entomobryidae). J. Agric. Univ. Puerto Rico 1979, 63, 214-222.

24. Absolon, K. Untersuchungen über Apterygoten auf Grund der Sammlungen des Wiener Hofmuseums. Ann. des K.K. Naturhist. Hofmus. 1903, 18, 91-111.

25. Mari-Mutt, J.A. A classification of the Orchesellinae with a key to tribes, genera and subgenera (Collembola: Entomobryidae). Ann. Entomol. Soc. Am. 1980, 73, 455-459. [CrossRef]

26. Zhang, F.; Deharveng, L. Systematic revision of Entomobryidae (Collembola) by integrating molecular and new morphological evidence. Zool. Scr. 2015, 44, 298-311. [CrossRef]

27. Soto-Adames, F.N.; Barra, J.A.; Christiansen, K.; Jordana, R. Suprageneric Classification of Collembola Entomobryomorpha. Ann. Entomol. Soc. Am. 2008, 101, 501-513. [CrossRef]

28. Nunes, R.C.; Santos-Costa, R.C.; Bellini, B.C. The first Neotropical Capbrya Barra, 1999 (Collembola: Orchesellidae: Nothobryinae) and the reinterpretation of Nothobryinae systematics. Zool. Anz. 2020, 288, 24-42. [CrossRef]

29. Arlé, R. Novas espécies de colêmbolas aquáticas (Nota preliminar). Atas Soc. Biol. Rio de Janeiro 1961, 5, 34-37.

30. Silveira, T.C.; Mendonça, M.C. New species of Nothobrya (Collembola: Entomobryidae) from Southeast Brazil. Zoologia 2016, 33, 1-6. [CrossRef]

31. Nunes, R.C.; Bellini, B.C. A new species of Nothobrya Arlé, 1961 (Collembola: Entomobryidae) from Brazil and notes on key characters for Nothobryinae taxonomy, with an identification key to the species of the subfamily. Zootaxa 2019, 4615, 375-391. [CrossRef]

32. Barra, J.A. Un nouveau genre Capbrya avec deux nouvelles espèces de la Province du Cap (Rép. Sud Africaine) (Collembola: Entomobryidae). Capbrya n. gen. d'Afrique du Sud (Insecta: Collembola). Bull. Inst. R. Sci. Nat. Belg. 1999, 69, 19-24. 
33. Jordana, R.; Arbea, J.I.; Simón, C.; Luciáñez, M.J. Fauna Iberica Volume 8: Collembola, Poduromorpha, 1st ed.; Museo Nacional de Ciencias Naturales, Consejo Superior de Investigaciones Científicas: Madrid, Spain, 1997; pp. 1-807.

34. Fjellberg, A. The Labial Palp in Collembola. Zool. Anz. 1999, 237, 309-330.

35. Gisin, H. Espèces nouvelles et lignées évolutives de Pseudosinella endogés. Mem. Est. Mus. Zool. Univ. Coimbra 1967, 301, 5-25.

36. Zhang, F.; Pan, Z.-X. Homology of labial chaetae in Entomobryoidea (Collembola). Zootaxa 2020, 4766, 498-500. [CrossRef]

37. Cipola, N.G.; Morais, J.W.; Bellini, B.C. A new species of Seira (Collembola: Entomobryidae: Seirini) from Northern Brazil, with the addition of new chaetotaxic characters. Zoologia 2014, 31, 489-495. [CrossRef]

38. Soto-Adames, F.N. Postembryonic development of the dorsal chaetotaxy in Seira dowlingi (Collembola, Entomobryidae); with an analysis of the diagnostic and phylogenetic significance of primary chaetotaxy in Seira. Zootaxa 2008, 1683, 1-31. [CrossRef]

39. Szeptycki, A. Morpho-systematic studies on Collembola. III. Body chaetotaxy in the first instars of several genera of the Entomobryomorpha. Acta Zool. Cracov. 1972, 17, 341-372.

40. Szeptycki, A. Chaetotaxy of the Entomobryidae and its Phylogenetical Significance. Morpho-Systematic Studies on Collembola; Polska Akademia Nauk: Kraków, Poland, 1979; Volume IV, pp. 1-219.

41. Zhang, F.; Yu, D.; Stevens, M.I.; Ding, Y. Colouration, chaetotaxy and molecular data provide species-level resolution in a species complex of Dicranocentrus (Collembola: Entomobryidae). Invertebr. Syst. 2018, 32, 1298-1315. [CrossRef]

42. Börner, C. Die Familien der Collembolen. Zool. Anz. 1913, 41, 315-322.

43. Xisto, T.; Mendonça, M.C. Contribuição ao conhecimento da morfologia e taxonomia de Dicranocentrus Schött, 1893, com histórico comentado para a Região Neotropical (Collembola: Entomobryidae). In Anais do III Simpósio de Entomologia do Rio de Janeiro, 1st ed.; Da-Silva, E.R., Passos, M.I.S., Aguiar, V.M., Lessa, C.S.S., Coelho, L.B.N., Eds.; Universidade Federal do Estado do Rio de Janeiro (UNIRIO): Rio de Janeiro, Brazil, 2016; pp. 151-165.

44. Mari-Mutt, J.A. A new species and complements to the descriptions of nine African or Oriental Dicranocentrus (Collembola: Entomobryidae). J. Agric. Univ. Puerto Rico 1981, 65, 160-170.

45. Mari-Mutt, J.A. New genus, a new species, and complements to the descriptions of seven Neotropical Dicranocentrus (Collembola: Entomobryidae: Orchesellinae). J. Agric. Univ. Puerto Rico 1981, 65, 90-107.

46. Kottek, M.; Grieser, J.; Beck, C.; Rudolf, B.; Rubel, F. World map of the Köppen-Geiger climate classification updated. Meteorol. Z. 2006, 15, 259-263. [CrossRef]

47. Mari-Mutt, J.A. Four new species of Dicranocentrus and Heteromurus from the State of Mérida, Venezuela (Collembola: Entomobryidae). J. Agric. Univ. Puerto Rico 1983, 67, 148-164.

48. Soto-Adames, F.N.; Anderson, E.W. Two new species and new records of springtails (Collembola: Entomobryidae, Paronellidae) from Nevis, Lesser Antilles. Fla. Entomol. 2017, 100, 32-41. [CrossRef]

49. Wray, D.L. New Collembola from Puerto Rico. J. Agric. Univ. Puerto Rico 1953, 37, 140-150.

50. Mari-Mutt, J.A. Eight new species of Dicranocentrus and redescriptions for D. thaicus and D. pilosus (Collembola: Entomobryidae: Orchesellinae). J. Agric. Univ. Puerto Rico 1985, 69, 297-322.

51. Bonet, F. Sur quelques Collemboles de l'Inde. Eos Rev. Esp. Entomol. 1930, 6, 249-273.

52. Uchida, H. Collembola Von Mikronisien. Bull. Tokyo Sci. Mus. 1944, 17, 1-23.

53. Mari-Mutt, J.A.; Bhattacharjee, R.K. Four new species of Dicranocentrus from Northeast India and Nepal. Pac. Insects 1980, 22, 162-170.

54. Ma, Y.; Chen, J.-X. A new Dicranocentrus species (Collembola: Entomobryidae) from China with a key to all species in the genus from Asia. Zootaxa 2007, 1633, 63-68. [CrossRef]

55. Schött, H. Kamerunische Collembolen. Linköping 1927, 5, 1-40.

56. Yoshii, R.; Suhardjono, Y.R. Notes on the Collembolan Fauna of Indonesia and its vicinities. I. Miscellaneous Notes, with special references to Seirini and Lepidocyrtini. Acta Zool. Asiae Orient. 1989, 1, 23-90.

57. Xu, G.-L.; Zhang, F. A new species of Dicranocentrus from China with comments on the systematic position of the genus. ZooKeys 2014, 417, 1-8. [CrossRef]

58. Wahlgren, E. Übersicht der aus dem Kilimandjaro-Mern-Gebiete bisher bekannten Collembolen. Sjöstedts Kilimandjaro-Meru Exp. 1908, 18, 1-10. 
59. Yosii, R. Studies on the Collembolan Fauna of Malay and Singapore with special reference to the genera: Lobella, Lepidocyrtus and Callyntrura. Contrib. Biol. Lab. Kyoto Univ. 1959, 10, 1-65.

60. Yosii, R. On some Collembola of Afghanistan, India and Ceylon, collected by the Kuphe-Expedition, 1960. R. Kyoto U. Sci. Exp. Karakoram Hindukush 1955 1966, 8, 333-405.

61. Cassagnau, P. Collemboles d'Amérique du Sud, II. Orchesellini, Paronellinae, Cyphoderinae. Biol. Amérique Australe 1963, 2, 127-148.

62. Bellini, B.C. Fauna de Collembola (Arthropoda) em áreas úmidas do semiárido. In Artrópodes do Semiárido: Biodiversidade e Conservação, 1st ed.; Bravo, F., Calor, A., Eds.; Printmídia: Feira de Santana, Brazil, 2014; pp. 57-68.

63. Abrantes, E.A.; Bellini, B.C.; Bernardo, A.N.; Fernandes, L.H.; Mendonça, M.C.; Oliveira, E.P.; Queiroz, G.C.; Sautter, K.D.; Silveira, T.C.; Zeppelini, D. Errata Corrigenda and update for the "Synthesis of Brazilian Collembola: An update to the species list." ABRANTES et al. (2010) Zootaxa, 2388: 1-22. Zootaxa 2012, 3168, 1-21. [CrossRef]

64. Bellini, B.C.; Godeiro, N.N. Novos registros de Collembola (Arthropoda, Hexapoda) para áreas úmidas do semiárido do Brasil. In Artrópodes do Semiárido II: Biodiversidade e conservação, 1st ed.; Bravo, F., Ed.; Métis: São Paulo, Brazil, 2017; pp. 28-53.

65. Denis, J.R. Contributo alla conoscenza del "Microgenton" di Costa Rica. II -Collemboles de Costa Rica avec une contribution au species de l'ordre. Boll. Lab. Entomol. Agric. 1931, 25, 69-170.

66. Bonet, F. Colémbolos de La República Argentina. Eos Rev. Esp. de Entomol. 1934, 9, 123-194.

67. Bellini, B.C.; Cipola, N.G. The Neotropical genera of Paronellinae (Collembola, Entomobryoidea, Paronellidae) with description of two new species and redescription of Campylothorax mitrai. Zootaxa 2017, 4300, 151-179. [CrossRef]

68. Prabhoo, N.R. Soil and litter Collembola of South India I-Arthropleona. Orient. Insects 1971, 5, 1-46. [CrossRef]

69. Yosii, R. Collembola of Khumbu Himal. Khumbu Himal 1971, 4, 80-130.

70. Mandal, G.P. A new genus of Heteromurini (Collembola: Entomobryidae) with dental base falcate macrochaetae, from India. Halteres 2018, 9, 74-85.

71. Christiansen, K.A.; Bellinger, P. Biogeography of Hawaiian Collembola: The simple principles and complex reality. Orient. Insects 1994, 28, 309-351. [CrossRef]

72. Zhang, F.; Sun, D.; Yu, D.; Wang, B. Molecular phylogeny supports S-chaetae as a key character better than jumping organs and body scales in classification of Entomobryoidea (Collembola). Sci. Rep. 2015, 5, 12471. [CrossRef]

73. Zhang, F.; Pan, Z.; Wu, J.; Ding, Y.; Yu, D.; Wang, B. Dental scales could occur in all scaled subfamilies of Entomobryidae (Collembola): New definition of Entomobryinae with description of a new genus and three new species. Invertebr. Syst. 2016, 30, 598-615. [CrossRef]

74. Zhang, F.; Ma, Y.; Greenslade, P. New Australian Paronellidae (Collembola) reveal anomalies in existing tribal diagnoses. Invertebr. Syst. 2017, 31, 375-393. [CrossRef]

75. Nunes, R.C.; Godeiro, N.N.; Pacheco, G.; Liu, S.; Gilbert, M.T.P.; Alvarez-Valin, F.; Zhang, F.; Bellini, B.C. The discovery of Neotropical Lepidosira (Collembola, Entomobryidae) and its systematic position. Zool. Scr. 2019, 48, 783-800. [CrossRef]

76. Santos, N.M.C.; Santos-Costa, R.C.; Siqueira, O.J.R.; Godeiro, N.N.; Bellini, B.C. Two new species of Entomobrya Rondani (Collembola, Entomobryidae) from northeastern Brazil and comments on the genus. Zootaxa 2020, 4731, 43-62. [CrossRef]

77. Godeiro, N.N.; Pacheco, G.; Liu, S.; Cipola, N.G.; Berbel-Filho, W.M.; Zhang, F.; Gilbert, M.T.P.; Bellini, B.C. Phylogeny of Neotropical Seirinae (Collembola, Entomobryidae) based on mitochondrial genomes. Zool. Scr. 2020, 49, 329-339. [CrossRef]

(C) 2020 by the authors. Licensee MDPI, Basel, Switzerland. This article is an open access article distributed under the terms and conditions of the Creative Commons Attribution (CC BY) license (http://creativecommons.org/licenses/by/4.0/). 